Article

\title{
Yuccalechins A-C from the Yucca schidigera Roezl ex Ortgies Bark: Elucidation of the Relative and Absolute Configurations of Three New Spirobiflavonoids and Their Cholinesterase Inhibitory Activities
}

\author{
Lukasz Pecio ${ }^{1, *,+}{ }^{\mathbb{D}}$, Mostafa Alilou ${ }^{2, *},+\left(\mathbb{D}\right.$, Solomiia Kozachok ${ }^{1}\left(\mathbb{D}\right.$, Ilkay Erdogan Orhan ${ }^{3}$, \\ Gokcen Eren $\left.{ }^{4}{ }^{(}\right)$, Fatma Sezer Senol Deniz ${ }^{3}$, Hermann Stuppner ${ }^{2}$ and Wiesław Oleszek ${ }^{1}$ (D) \\ 1 Department of Biochemistry and Crop Quality, Institute of Soil Science and Plant Cultivation-State Research \\ Institute, Czartoryskich 8, 24-100 Puławy, Poland; skozachok@iung.pulawy.pl (S.K.); \\ wieslaw.oleszek@iung.pulawy.pl (W.O.) \\ 2 Institute of Pharmacy/Pharmacognosy, Center for Molecular Biosciences Innsbruck, University of Innsbruck, \\ Innrain 80/82, Innsbruck 6020, Austria; hermann.stuppner@uibk.ac.at \\ 3 Department of Pharmacognosy, Faculty of Pharmacy, Gazi University, 06330 Ankara, Turkey; \\ iorhan@gazi.edu.tr (I.E.O.); fssenol@gazi.edu.tr (F.S.S.D.) \\ 4 Department of Pharmaceutical Chemistry, Faculty of Pharmacy, Gazi University, 06330 Ankara, Turkey; \\ gokcene@gazi.edu.tr \\ * Correspondence: lpecio@iung.pulawy.pl (Ł.P.); mostafa.alilou@student.uibk.ac.at (M.A.); \\ Tel.: +48-814-786-882 (Ł.P.); +43-512-507-58437 (M.A.) \\ + These authors contributed equally to this work.
}

Academic Editor: Wolfgang Robien

Received: 30 October 2019; Accepted: 14 November 2019; Published: 16 November 2019

\begin{abstract}
The ethyl acetate fraction of the methanolic extract of Yucca schidigera Roezl ex Ortgies bark exhibited moderate acetylcholinesterase $(\mathrm{AChE})$ and butyrylcholinesterase $(\mathrm{BChE})$ inhibitory activity (IC 5047.44 and $47.40 \mu \mathrm{g} \mathrm{mL}{ }^{-1}$, respectively). Gel filtration on Sephadex LH-20 and further RP-C 18 preparative HPLC of EtOAc fraction afforded 15 known and 3 new compounds, stereoisomers of larixinol. The structures of the isolated spirobiflavonoids 15, 26, and 29 were elucidated using 1D and 2D NMR and MS spectroscopic techniques. The relative configuration of isolated compounds was assigned based on coupling constants and ROESY (rotating-frame Overhauser spectroscopy) correlations along with applying the DP4+ probability method in case of ambiguous chiral centers. Determination of absolute configuration was performed by comparing calculated electronic circular dichroism (ECD) spectra with experimental ones. Compounds 26 and 29, obtained in sufficient amounts, were evaluated for activities against $\mathrm{AChE}$ and $\mathrm{BChE}$, and they showed a weak inhibition only towards $\mathrm{AChE}$ ( $\mathrm{IC}_{50} 294.18 \mu \mathrm{M}$ for $\mathbf{2 6}$, and $655.18 \mu \mathrm{M}$ for 29). Furthermore, molecular docking simulations were performed to investigate the possible binding modes of $\mathbf{2 6}$ and $\mathbf{2 9}$ with AChE.
\end{abstract}

Keywords: Yucca schidigera; Asparagaceae; spirobiflavonoid; absolute configuration; DP4+; ECD; Alzheimer's disease

\section{Introduction}

Yucca schidigera Roezl ex Ortgies (syn. Yucca mohavensis Sargent) belonging to the Asparagaceae family, is a small evergreen tree (up to $5 \mathrm{~m}$ tall), distributed from Southern Nevada to Mexico (Baja California) [1]. Native Americans used the plant for food and fiber, and its extracts have been used for centuries in folk medicine to treat a wide variety of inflammatory disorders, headaches, 
arthritis, rheumatism, and bacterial infections (gonorrhea) [2], but nowadays it is mostly used in cosmetics, the beverage industry, as foaming agent in soft drinks, and as a food supplement in the form of condensed yucca syrup [3]. The syrup is obtained from mechanically pressed logs of yucca, and steroidal glycosides (furostanol- and spirostanol-type) with middle- and short-length saccharide chains are its predominant constituents [4,5], while the bark contains more polar, bidesmosidic saponins [6]. These products possess the generally recognized as safe (GRAS) label given by the FDA, which allows human dietary use. However, there are several publications mentioning a wide variety of phenolic compounds present in the bark of the plant (the waste) byproducts of $Y$. schidigera in commercial applications [7-10]. Among them, derivatives of trans-resveratrol (trans-3,4',5-trihydroxystilbene) and trans-3,3',5,5'-tetrahydroxy-4'-methoxystilbene, such as yuccaols A-E and yuccaone A, which are unique spiro-compounds including $C_{15}$ and $C_{14}$ units condensed to form a $\gamma$-lactone ring, very rarely occur in the plant kingdom [11]. These compounds are known for their various antioxidant, radical scavenging, inhibiting iNOS expression, and platelet aggregation activities in vitro [7,12]. At first, yuccaols A-E and yuccaone A were isolated from Y. schidigera bark [7,9]. Lately, yuccaols C-E have been identified in roots of Y. gloriosa L. [13] and yuccaols C and D in Y. pringlei Greenm [14]. Similarly to yuccaols, gloriosaols A-E [13] and yuccalides A-C [15] possess spiro-structures and have been isolated from Y. gloriosa roots. Spirobiflavonoids also belong to the group mentioned, and the first compound of this class, named larixinol, was identified in 1986 in the bark of Larix gmelini [16]. Two spirobiflavonoids, daphnodorins $\mathrm{M}$ and $\mathrm{N}$, were isolated from the roots and the bark of Daphne acutiloba [17]. Later, another four new spirobiflavonoids, named olgensisinols A-D, along with a known one, vitisinol, were isolated from the stem bark of L. olgensis HENRY var. koreana NAKAI [18], two new spirobiflavonoids from Abies chensiensis (3-epi-larixinol and 3,2'-epi-larixinol) [19], and six from A. sachalinensis [20]. By 2010, only 16 compounds of this class were identified in plants [11,21].

Simultaneously, dementia is a growing problem in public health as the elderly form a higher proportion of the population. It is estimated that by 2050, over 100 million people worldwide will suffer from this disease [22]. The most common form of dementia in the elderly (ages 65-90) is Alzheimer's disease (AD) [23]. It is, thus, no surprise that a large volume of research is being directed at understanding the causes of and potential treatments for AD. Although the etiology of AD has not been fully elucidated yet, modern treatment strategies typically comprise anticholinesterases, including acetylcholinesterase (AChE) (EC 3.1.1.7) and butyrylcholinesterase (BChE) (EC 3.1.1.8); antioxidants; $\alpha, \beta$, and $\gamma$ secretase inhibitors; $N$-methyl-D-aspartate receptor agonists [24]; and inhibitors of the phosphorylation of AD-associated protein [25]. Given the complexity of the disease, other mechanisms are also involved in AD development, such as oxidative stress, neuroinflammation, excitotoxicity, metal dyshomeostasis, and mitochondrial damage [26]. However, there is abundant evidence that defects in cholinergic synaptic transmission, in particular nicotinic acetylcholine receptor (nAChR)-mediated signaling, plays a major role. The most remarkable biochemical change in AD patients is a reduction of acetylcholine levels in the hippocampus and cortex of the brain. Thus, inhibition of AChE and BChE is the major approach for treating $\mathrm{AD}[27,28]$. Over recent decades, several pharmaceuticals have become available on the market for clinical use; however, none of them has the ability to discontinue the disease. This is why there is still a great need for the discovery of new drug candidates of natural and synthetic origin with multitarget activities to treat AD [24,25]. There are numerous AChE/BChE inhibitors (AChEi/BChEi) of natural and semi-synthetic origin identified, most of which belong to the alkaloid, terpenoid, and phenolic groups of natural products [25]. The oldest known, discovered in 1846 , is a highly toxic parasympathomimetic derivative of indole: physostigmine. There are many natural and synthetic derivatives of indole, many times patented as AChEi [28]. Another alkaloid, galantamine, belonging to the isoquinoline group of compounds, was approved in 2001 as AChEi [29]. Huperzine A, a sesquiterpene alkaloid isolated in 1986 from Lycopodium serratum Thunb., was approved in China in 1990 as an anti-Alzheimer drug, and was patented and distributed in the USA as a dietary supplement [30]. 
A great number of phenolic compounds have been identified as candidates for $\mathrm{AD}$ treatment $[25,31,32]$. They constitute of one of the widest chemical classes amongst plant secondary metabolites. To date, phenolic substances have been identified with many pharmacological effects including antioxidant, anti-inflammatory, antimutagenic, chemopreventive, anticancer, and antiviral activities. Some plant phenolics have been demonstrated to inhibit both $\mathrm{AChE}$ and $\mathrm{BChE}$ to varying extents. Most of these studies focused on in vitro tests, and only few studies were performed on insects, tissue, and animal models, with rarely any clinical studies [33]. Phenolics, besides their AChE and BChE inhibitory activities, also have very important antioxidant activity, which may enhance their protective effects. It has been proven that oxidative stress caused by reactive oxygen species (ROS) is involved in the aging processes. It has been suggested that free radicals damage mitochondria in certain areas of the brain that are particularly important for memory and cognitive processes and are associated with the pathogenesis of AD [34-36]. Hence, supplementation of the diet with antioxidants in people may reduce the risk of $\mathrm{AD}$ [34]. This was a major point of a number of studies performed on plants with high antioxidant potential [37]. Moreover, numerous reports indicated multitarget effects of resveratrol on AD [25]. Resveratrol oligomers showed a significant AChE/BChE inhibitory activity [38], and it was suggested to be used as a starting compound in the design of multitargeted drugs for the treatment of AD [39]. The diverse biological effects of the constituents of Y. schidigera bark encouraged us to investigate further the structurally related compounds using modern chromatographic and spectroscopic techniques.

Furthermore, as nature possesses the ability to create innumerable complex chemical structures, very often with chiral properties [40,41], the need to properly assign stereochemistry of natural products has emerged [42,43]. The use of quantum chemical calculations and computer-assisted structure elucidation (CASE) methods in solving structural validation problems simplified this task and reduced the risk of misinterpretations [44,45]. DP4-based nuclear magnetic resonance (NMR) chemical shift calculation is one of the most advanced approaches for stereochemical assignments of organic molecules when only one set of experimental data is available [46]. This method implements gauge-independent atomic orbital (GIAO) NMR for chemical shift calculations of geometries obtained by Merck molecular force fields (MMFFs) [47-50]. As it failed in assignment of some challenging molecules, Grimblat et al. [51] developed a DP4+ probability-based chemical shift analysis, where using B3LYP/6-31+G** geometries and adding the unscaled shift values significantly increased the performance of the method, which led to more accurate and confident results in establishing the stereochemistry of challenging isomeric compounds. However, relying solely on this approach often is not sufficient in determining the absolute configuration (AC) of natural compounds. One of the approaches, when the chiral compound possesses an appropriate chromophore, is the use of electronic circular dichroism (ECD) by comparing the experimental spectrum with the one calculated by time-dependent density functional theory (TDDFT) [52-54]. This, along with the careful study of nuclear Overhauser effects (NOEs) observed in the NMR spectra and using $\mathrm{H}-\mathrm{H}$ or $\mathrm{C}-\mathrm{H}$ coupling constants, provides an unambiguous tool for assignment of the AC.

Thus, the aim of this work was to study numerous phenolics of the plant, evaluate the $\mathrm{AChE} / \mathrm{BChE}$ inhibitory activity of newly isolated compounds, and to assign their stereochemistry and absolute configurations using DP4+ probability-based chemical shift analysis and quantum chemical calculation methods.

\section{Results}

\subsection{Identification of the Constituents Found in the Y. schidigera Ethyl Acetate Fraction}

Initial chromatographic ultra-high-performance liquid chromatography-charged aerosol detector-mass spectrometry (UHPLC-CAD-MS) analysis of the ethyl acetate fraction of the methanolic extract obtained from $Y$. schidigera bark revealed the presence of numerous (over 70) peaks, tentatively identified as phenolic acids $(2,4,5)$, flavan-3-ols $(7)$, flavanonols $(\mathbf{1 2}, \mathbf{1 6})$, flavonols $(\mathbf{2 0}, \mathbf{2 3}, \mathbf{3 1}, \mathbf{4 4})$, 
flavanones (38), stilbenoids $(\mathbf{1 3}, \mathbf{2 1})$, spirobiflavonoids $(\mathbf{1 5}, \mathbf{2 6}, \mathbf{2 9}, \mathbf{3 0}, 36)$, yuccaols $(37,39,42,47$, 48), yuccalide A (40), and gloriosaols (46, 49, 50, 52, 54, 58, 60) (Figure 1, Table 1, peak numbers assigned based on retention times). Their identity was confirmed by the application of accurate mass measurements, MS/MS fragmentation patterns, ultraviolet-visible (UV-vis) spectra, and by comparison with existing literature data. To ensure the identity of the main fraction components, a multistep purification procedure was applied and led to the isolation of 18 compounds (Figure 2), which were further analyzed using various spectral techniques: $1 \mathrm{D}$ and 2D NMR, quadrupole time-of-flight-high-resolution electrospray ionization mass spectrometry (QTOF-HRESIMS), optical rotations, ECD, and, for compounds 15, 26, 29, calculation of both ECD and NMR spectra. As a result, we isolated three new compounds and confirmed the presence of aromadendrin, naringenin, resveratrol, trans-3,3',5,5'-tetrahydroxy-4'-methoxystilbene, yuccaols A-E, yuccalide A, and surprisingly, gloriosaols A and C-E, previously reported in roots of Y. gloriosa [13,55].

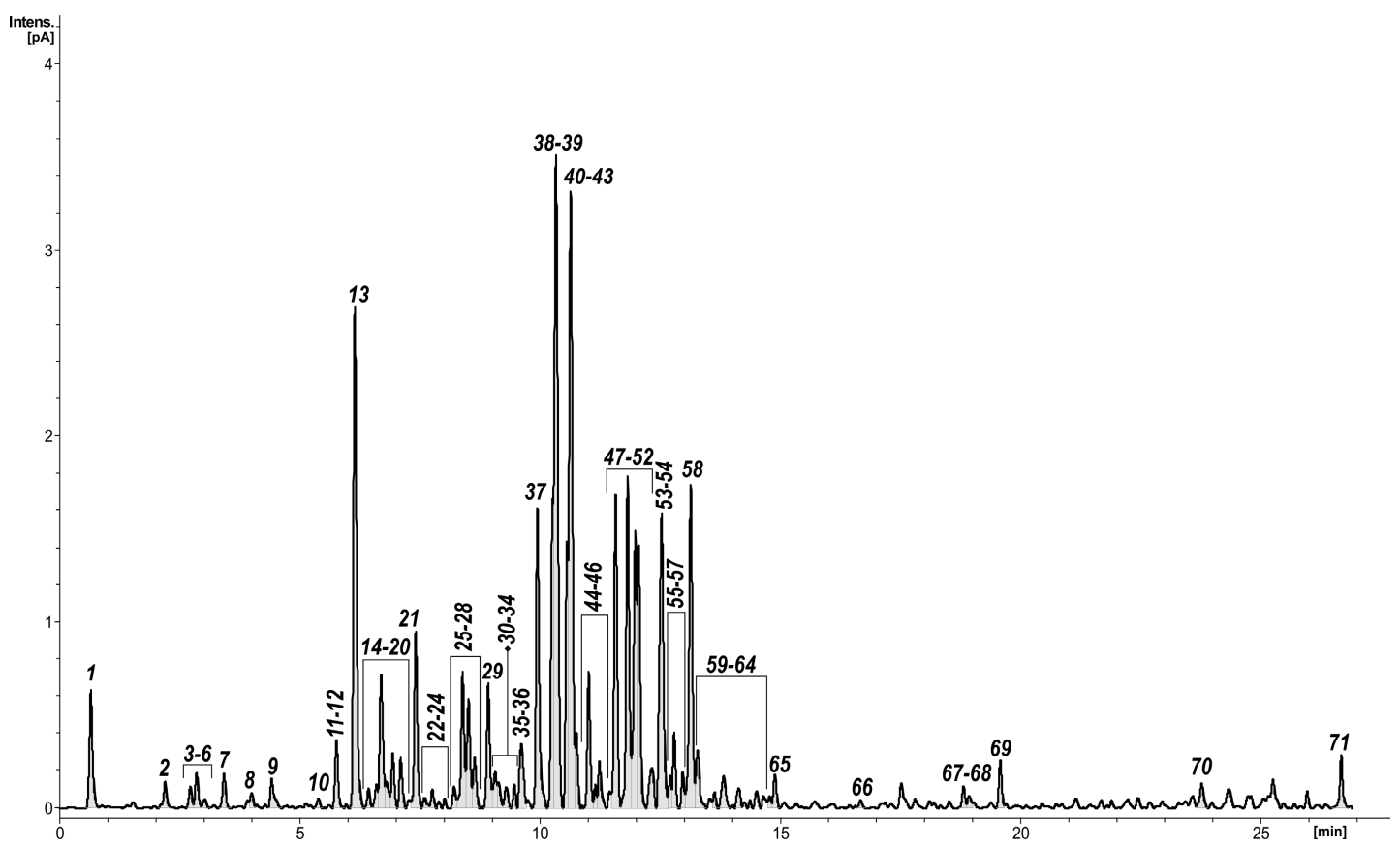

Figure 1. Ultra-high-performance liquid chromatography-charged aerosol detector (UHPLC-CAD) profile of the Yucca schidigera ethyl acetate fraction. 
Table 1. Compounds identified in the $Y$. schidigera ethyl acetate fraction using ultra-high-performance liquid chromatography-quadrupole time-of-flight-tandem mass spectrometry (UHPLC-QTOF-MS/MS)

\begin{tabular}{|c|c|c|c|c|c|c|c|c|c|}
\hline No. & Compound Name & $\mathrm{RT}(\min )$ & $\lambda_{\max }(\mathrm{nm})$ & $\begin{array}{l}\text { Neutral } \\
\text { Formula }\end{array}$ & Error * $(\mathrm{ppm})$ & $\mathbf{M} \sigma^{* *}$ & $\begin{array}{l}\text { Observed } \\
{[\mathrm{M}-\mathrm{H}]^{-}}\end{array}$ & Major Fragments (\%) & Reference \\
\hline 1. & Mixture of polar compounds & 0.66 & - & - & - & - & - & & - \\
\hline 2. & $p$-Hydroxybenzoic acid & 2.20 & 255 & $\mathrm{C}_{7} \mathrm{H}_{6} \mathrm{O}_{3}$ & 1.5 & 1.4 & 137.0242 & $137.0243(100), 93.0342(69)$ & [56] \\
\hline 3. & Hydroxymethoxyacetophenone & 2.73 & - & $\mathrm{C}_{9} \mathrm{H}_{10} \mathrm{O}_{3}$ & 2.2 & 6.2 & 165.0554 & $165.0553(100), 123.0450(25), 152.0112(6)$ & [57] \\
\hline 4. & Hydroxybenzylmalic acid & 2.73 & - & $\mathrm{C}_{11} \mathrm{H}_{12} \mathrm{O}_{6}$ & 2.2 & 1.9 & 239.0556 & $\begin{array}{c}179.0347(100), 239.0557(77), 177.0553(55), \\
149.0604(32), 133.0656(11), 195.0659(11)\end{array}$ & [58] \\
\hline 5. & Caffeic acid & 2.86 & 215,323 & $\mathrm{C}_{9} \mathrm{H}_{8} \mathrm{O}_{4}$ & 2.3 & 0.7 & 179.0346 & $135.0449(100), 179.0347(65)$ & [56] \\
\hline 6. & Unknown & 3.02 & - & $\mathrm{C}_{23} \mathrm{H}_{18} \mathrm{O}_{10}$ & 0.9 & 20.4 & 453.0823 & $\begin{array}{c}310.0484(100), 325.0716(49), 376.0585(48) \\
256.0372(39)\end{array}$ & \\
\hline 7. & (Epi)afzelechin & 3.43 & - & $\mathrm{C}_{15} \mathrm{H}_{14} \mathrm{O}_{5}$ & 1.1 & 1.4 & 273.0766 & $\begin{array}{c}273.0766(100), 205.0867(39), 255.0660(29), \\
137.0247(29), 229.0868(28)\end{array}$ & [11] \\
\hline 8. & Unknown & 4.00 & - & $\mathrm{C}_{22} \mathrm{H}_{16} \mathrm{O}_{8}$ & -0.1 & 17.4 & 407.0773 & $\begin{array}{c}345.0768(100), 163.0037(92), 269.0455(80), \\
293.0819(68), 279.0661(67)\end{array}$ & - \\
\hline 9. & Unknown & 4.42 & - & $\mathrm{C}_{29} \mathrm{H}_{22} \mathrm{O}_{9}$ & 0.7 & 9.6 & 513.1187 & $\begin{array}{c}293.0817(100), 379.0823(70), 275.0713(65), \\
335.0924(61), 361.0721(59)\end{array}$ & - \\
\hline 10. & Unknown & 5.39 & - & $\mathrm{C}_{20} \mathrm{H}_{24} \mathrm{O}_{7}$ & 0.2 & 23.0 & 375.1448 & $\begin{array}{c}297.1133(100), 282.0900(28), 151.0401(22), \\
315.1256(9), 136.0186(6)\end{array}$ & - \\
\hline 11. & Unknown & 5.77 & 323 & $\mathrm{C}_{14} \mathrm{H}_{12} \mathrm{O}_{4}$ & -0.3 & 1.4 & 243.0663 & 243.0661 (100), 201.0554 (7), 225.0558 (3) & - \\
\hline 12. & Dihydrorobinetin & 5.77 & 263 & $\mathrm{C}_{15} \mathrm{H}_{12} \mathrm{O}_{7}$ & -0.3 & 0.9 & 303.0511 & $\begin{array}{c}183.0299(100), 139.0402(67), 165.0195(20), \\
137.0246(9), 97.0294(4), 95.0500(3)\end{array}$ & [59] \\
\hline 13. & $\begin{array}{l}\text { Trans }-3,3^{\prime}, 5,5,5^{\prime} \text {-tetrahydroxy-4'- } \\
\text { methoxystilbene }\end{array}$ & 6.15 & 227,315 & $\mathrm{C}_{15} \mathrm{H}_{14} \mathrm{O}_{5}$ & 1.0 & 2.6 & 273.0766 & $\begin{array}{c}258.0526(100), 240.0434(9), 273.0766(5) \\
196.0528(4), 188.0477(2), 172.0532(2)\end{array}$ & {$[8,9]$} \\
\hline 14. & Unknown & 6.43 & - & $\mathrm{C}_{30} \mathrm{H}_{24} \mathrm{O}_{11}$ & 2.4 & 18.5 & 559.1232 & $\begin{array}{c}373.0715(100), 433.0922(42), 418.0688(32), \\
401.0657(22), 125.0245(16)\end{array}$ & - \\
\hline 15. & Yuccalechin A & 6.60 & 215 & $\mathrm{C}_{30} \mathrm{H}_{22} \mathrm{O}_{10}$ & 1.8 & 6.0 & 541.1131 & $\begin{array}{c}308.0346(100), 281.0468(88), 267.0313(60), \\
269.0453(41), 415.0843(30)\end{array}$ & - \\
\hline 16. & $\begin{array}{c}\text { Aromadendrin } \\
\text { (dihydrokaempferol) }\end{array}$ & 6.70 & 295 & $\mathrm{C}_{15} \mathrm{H}_{12} \mathrm{O}_{6}$ & 1.3 & 4.8 & 287.0557 & $\begin{array}{c}259.0608(100), 125.0243(68), 287.0243(39), \\
243.0658(25), 201.0554(14)\end{array}$ & {$[60]$} \\
\hline 17. & Unknown & 6.81 & - & $\mathrm{C}_{29} \mathrm{H}_{24} \mathrm{O}_{10}$ & 1.9 & 25.9 & 531.1286 & $\begin{array}{c}257.0459 \text { (100), } 393.0605(65), 531.1293(64), \\
378.0752 \text { (45), } 269.0489(40)\end{array}$ & - \\
\hline 18. & Glyceryl azelate & 6.81 & - & $\mathrm{C}_{12} \mathrm{H}_{22} \mathrm{O}_{6}$ & 1.3 & 11.9 & 261.1340 & $\begin{array}{c}187.0974(100), 125.0971(31), 261.1342(15), \\
169.0869(13)\end{array}$ & {$[61]$} \\
\hline 19. & Azelaic acid & 6.94 & - & $\mathrm{C}_{9} \mathrm{H}_{16} \mathrm{O}_{4}$ & 1.8 & 0.4 & 187.0972 & $\begin{array}{c}187.0973(100), 125.0970(73), 169.0868(20), \\
97.0655(3)\end{array}$ & [62] \\
\hline 20. & Myricetin & 7.10 & 370 & $\mathrm{C}_{15} \mathrm{H}_{10} \mathrm{O}_{8}$ & 0.8 & 1.4 & 317.0300 & $\begin{array}{c}317.0301 \text { (100), } 178.9983(59), 151.0035(42) \\
137.0244(19)\end{array}$ & [60] \\
\hline 21. & Trans-resveratrol & 7.42 & 215,307 & $\mathrm{C}_{14} \mathrm{H}_{12} \mathrm{O}_{3}$ & 3.3 & 4.5 & 227.0706 & 227.0707 (100), $185.0603(8), 183.0809$ (2) & {$[9,63]$} \\
\hline 22. & Oxododecanedioic acid & 7.60 & - & $\mathrm{C}_{12} \mathrm{H}_{20} \mathrm{O}_{5}$ & 2.9 & 10.7 & 243.1231 & $\begin{array}{c}225.1124(100), 243.1232(45), 181.1230(24), \\
207.1021(20), 199.1335(17)\end{array}$ & - \\
\hline 23. & Tetrahydroxyflavone & 7.60 & - & $\mathrm{C}_{15} \mathrm{H}_{10} \mathrm{O}_{6}$ & 2.2 & 14.5 & 285.0398 & $\begin{array}{c}285.0400(100), 151.0033(91), 257.0450(55), \\
107.0137(15), 213.0545(11)\end{array}$ & {$[64]$} \\
\hline 24. & Unknown & 7.77 & - & $\mathrm{C}_{28} \mathrm{H}_{22} \mathrm{O}_{8}$ & 2.5 & 5.6 & 485.1230 & $\begin{array}{c}257.0451(100), 485.1227(61), 391.0819(35), \\
347.0921(34), 227.0706(24)\end{array}$ & - \\
\hline
\end{tabular}


Table 1. Cont

\begin{tabular}{|c|c|c|c|c|c|c|c|c|c|}
\hline No. & Compound Name & RT (min) & $\lambda_{\max }(\mathrm{nm})$ & $\begin{array}{c}\text { Neutral } \\
\text { Formula }\end{array}$ & Error $^{*}(\mathrm{ppm})$ & $\mathbf{M} \sigma^{* *}$ & $\begin{array}{l}\text { Observed } \\
{[\mathrm{M}-\mathrm{H}]^{-}}\end{array}$ & Major Fragments (\%) & Reference \\
\hline 25. & Unknown & 8.21 & - & $\mathrm{C}_{20} \mathrm{H}_{26} \mathrm{O}_{6}$ & 3.0 & 4.8 & 361.1646 & $\begin{array}{c}361.1645 \text { (100), } 346.1417(51), 165.0548(27) \\
179.0701(8), 122.0374(7)\end{array}$ & - \\
\hline 26. & Yuccalechin B & 8.39 & 210 & $\mathrm{C}_{30} \mathrm{H}_{22} \mathrm{O}_{10}$ & 3.1 & 16.5 & 541.1123 & $\begin{array}{c}308.0334(100), 281.0459(99), 267.0331(71), \\
415.0829(32), 361.0719(31)\end{array}$ & - \\
\hline 27. & Unknown & 8.51 & 319 & $\mathrm{C}_{29} \mathrm{H}_{24} \mathrm{O}_{19}$ & 3.6 & 1.9 & 515.1329 & $\begin{array}{c}362.0784(100), 515.1332 \text { (70), } 241.0500(59), \\
253.0507(42), 291.0664(19)\end{array}$ & - \\
\hline 28. & Unknown & 8.64 & 319 & $\mathrm{C}_{15} \mathrm{H}_{12} \mathrm{O}_{6}$ & 2.8 & 13.3 & 287.0553 & $\begin{array}{c}287.0553(100), 219.0656(25), 199.0423(16), \\
227.0354(11), 185.0238(8)\end{array}$ & - \\
\hline 29. & Yuccalechin C & 8.93 & 211 & $\mathrm{C}_{30} \mathrm{H}_{22} \mathrm{O}_{10}$ & 3.5 & 4.7 & 541.1121 & $\begin{array}{c}308.0337(100), 281.0459(93), 267.0306(43), \\
269.0449(39), 415.0828(30)\end{array}$ & - \\
\hline 30. & Larixinol isomer IV & 9.07 & - & $\mathrm{C}_{30} \mathrm{H}_{22} \mathrm{O}_{10}$ & 3.0 & 15.3 & 541.1124 & $\begin{array}{c}267.0300(100), 269.0450(71), 281.0463(62), \\
308.0339(48), 445.1277(46)\end{array}$ & - \\
\hline 31. & Quercetin & 9.14 & 219,367 & $\mathrm{C}_{15} \mathrm{H}_{10} \mathrm{O}_{7}$ & 2.7 & 3.0 & 301.0346 & $\begin{array}{c}301.0344(100), 151.0031 \text { (77), } 178.9979(51), \\
121.0292(10), 273.0401 \text { (10) }\end{array}$ & [64] \\
\hline 32. & Unknown & 9.30 & - & $\mathrm{C}_{30} \mathrm{H}_{22} \mathrm{O}_{10}$ & 3.6 & 6.7 & 541.1121 & $\begin{array}{c}269.0444(100), 390.0732(39), 362.0798(15), \\
308.0335(15), 281.0459(13)\end{array}$ & - \\
\hline 33. & Unknown & 9.30 & - & $\mathrm{C}_{16} \mathrm{H}_{12} \mathrm{O}_{8}$ & 2.7 & 14.8 & 331.0451 & $316.0214(100), 331.0448(9)$ & - \\
\hline 34. & Unknown & 9.48 & - & $\mathrm{C}_{15} \mathrm{H}_{12} \mathrm{O}_{5}$ & 3.3 & 2.1 & 271.0603 & $\begin{array}{c}253.0496 \text { (100), } 227.0704(52), 271.0602(26), \\
185.0598(4), 225.0551(3)\end{array}$ & - \\
\hline 35. & Unknown & 9.62 & 315 & $\mathrm{C}_{24} \mathrm{H}_{20} \mathrm{O}_{8}$ & 3.9 & 5.3 & 435.1068 & $\begin{array}{c}420.0834(100), 435.1069(96), 281.0462(62), \\
393.0964(50), 240.0420(31)\end{array}$ & - \\
\hline 36. & Larixinol isomer V & 9.62 & 315 & $\mathrm{C}_{30} \mathrm{H}_{22} \mathrm{O}_{10}$ & 3.4 & 4.1 & 541.1122 & $\begin{array}{c}308.0329(100), 281.0460(97), 267.0328(60) \\
415.0826(34), 497.1219(23)\end{array}$ & [20] \\
\hline 37. & Yuccaol E & 9.96 & 207,319 & $\mathrm{C}_{30} \mathrm{H}_{22} \mathrm{O}_{10}$ & 3.7 & 8.9 & 541.1120 & $\begin{array}{c}498.0936 \text { (100), } 513.1171(52), 267.0310(27), \\
429.0974(11), 375.0511 \text { (11) }\end{array}$ & {$[7,63]$} \\
\hline 38. & Naringenin & 10.27 & 211,291 & $\mathrm{C}_{15} \mathrm{H}_{12} \mathrm{O}_{5}$ & 3.5 & 1.6 & 271.0612 & $\begin{array}{c}271.0603(100), 151.0031(83), 119.0499(23), \\
177.0188(11), 107.0133(6)\end{array}$ & [64] \\
\hline 39. & Yuccaol C & 10.33 & 211,319 & $\mathrm{C}_{30} \mathrm{H}_{22} \mathrm{O}_{10}$ & 3.2 & 4.5 & 541.1123 & $\begin{array}{c}267.0303(100), 513.1171(65), 498.0934(63), \\
239.0348(36), 429.0981(23)\end{array}$ & {$[9,63,65]$} \\
\hline 40. & Yuccalide A & 10.57 & 211,323 & $\mathrm{C}_{30} \mathrm{H}_{22} \mathrm{O}_{10}$ & 4.9 & 12.0 & 541.1114 & $\begin{array}{c}498.0934(100), 267.0304(76), 513.1168(72), \\
239.0348(26), 429.0979(18)\end{array}$ & [15] \\
\hline 41. & Unknown & 10.57 & 211,323 & $\mathrm{C}_{29} \mathrm{H}_{20} \mathrm{O}_{9}$ & 3.6 & 8.9 & 511.1016 & $\begin{array}{c}267.0302(100), 483.1069(36), 239.0345(33), \\
385.0713(26), 399.0857(22)\end{array}$ & - \\
\hline 42. & Yuccaol D & 10.64 & 211,323 & $\mathrm{C}_{30} \mathrm{H}_{22} \mathrm{O}_{10}$ & 3.6 & 7.4 & 541.1121 & $\begin{array}{c}498.0934(100), 267.0303(89), 513.1168(77), \\
239.0348(30), 429.0979(21)\end{array}$ & {$[7,63,65]$} \\
\hline 43. & Unknown & 10.76 & 215 & $\mathrm{C}_{30} \mathrm{H}_{22} \mathrm{O}_{9}$ & 4.0 & 5.3 & 525.1170 & $\begin{array}{c}399.0868(100), 267.0300(84), 269.0443(66), \\
361.0695(42), 333.0766(38)\end{array}$ & - \\
\hline 44. & Kaempferol & 11.02 & 265,363 & $\mathrm{C}_{15} \mathrm{H}_{10} \mathrm{O}_{6}$ & 3.3 & 8.2 & 285.0395 & $285.0394(100)$ & [64] \\
\hline 45. & Unknown & 11.16 & - & $\mathrm{C}_{30} \mathrm{H}_{22} \mathrm{O}_{9}$ & 3.3 & 4.3 & 525.1174 & $\begin{array}{c}399.0865(100), 267.0310(72), 361.0707(60) \\
307.0607(38), 293.0445(36)\end{array}$ & - \\
\hline 46. & Gloriosaol isomer I & 11.24 & 320 & $\mathrm{C}_{45} \mathrm{H}_{30} \mathrm{O}_{15}$ & 3.6 & 22.9 & 809.1483 & $\begin{array}{c}267.0296(100), 239.0343(55), 211.0395(11), \\
513.1166(3), 541.1121(3)\end{array}$ & - \\
\hline 47. & Yuccaol A & 11.58 & 207,327 & $\mathrm{C}_{29} \mathrm{H}_{20} \mathrm{O}_{8}$ & 3.1 & 7.2 & 495.1070 & $\begin{array}{c}267.0310(100), 467.1120(67), 239.0347(60), \\
399.1224(21), 357.1122(16)\end{array}$ & {$[9,63,65]$} \\
\hline
\end{tabular}


Table 1. Cont.

\begin{tabular}{|c|c|c|c|c|c|c|c|c|c|}
\hline No. & Compound Name & RT (min) & $\lambda_{\max }(\mathrm{nm})$ & $\begin{array}{l}\text { Neutral } \\
\text { Formula }\end{array}$ & Error* ${ }^{*}(\mathrm{ppm})$ & $M \sigma * *$ & $\begin{array}{l}\text { Observed } \\
{[\mathrm{M}-\mathrm{H}]^{-}}\end{array}$ & Major Fragments (\%) & Reference \\
\hline 48. & Yuccaol B & 11.83 & 207,327 & $\mathrm{C}_{29} \mathrm{H}_{20} \mathrm{O}_{8}$ & 2.6 & 9.2 & 495.1073 & $\begin{array}{c}267.0312(100), 239.0349(50), 467.1122(44), \\
399.1229(16), 357.1124(12)\end{array}$ & {$[9,63,65]$} \\
\hline 49. & Gloriosaol E & 11.98 & 207,323 & $\mathrm{C}_{45} \mathrm{H}_{30} \mathrm{O}_{15}$ & 3.8 & 12.0 & 809.1481 & $\begin{array}{c}267.0294(100), 239.0343(44), 211.0395(8), \\
541.1118(3), 513.1171(3)\end{array}$ & {$[13,65]$} \\
\hline 50. & Gloriosaol D & 12.05 & 207,323 & $\mathrm{C}_{45} \mathrm{H}_{30} \mathrm{O}_{15}$ & 3.6 & 7.3 & 809.1483 & $\begin{array}{c}267.0294(100), 239.0343(45), 211.0395(9) \\
541.1117(3), 513.1171(3)\end{array}$ & {$[13,65]$} \\
\hline 51. & Unknown & 12.33 & - & $\mathrm{C}_{30} \mathrm{H}_{22} \mathrm{O}_{9}$ & 3.1 & 5.3 & 525.1175 & $\begin{array}{c}267.0314(100), 497.1226(94), 239.0359(41), \\
413.1018(29), 482.0994(25)\end{array}$ & - \\
\hline 52. & Gloriosaol isomer II & 12.33 & - & $\mathrm{C}_{45} \mathrm{H}_{30} \mathrm{O}_{15}$ & 3.3 & 14.3 & 809.1485 & $\begin{array}{c}267.0297(100), 239.0345(43), 211.0396(9) \\
541.1125(4), 513.1166(3)\end{array}$ & - \\
\hline 53. & Unknown & 12.53 & 283 & $\mathrm{C}_{16} \mathrm{H}_{16} \mathrm{O}_{3}$ & 2.7 & 15.0 & 255.1020 & $255.1020(100)$ & - \\
\hline 54. & Gloriosaol A & 12.53 & 207,319 & $\mathrm{C}_{45} \mathrm{H}_{30} \mathrm{O}_{15}$ & 3.3 & 3.9 & 809.1485 & $\begin{array}{c}267.0295(100), 239.0343(49), 211.0395(10), \\
541.1117(4), 513.1172(3)\end{array}$ & {$[55,65]$} \\
\hline 55. & Trihydroxyoctadecadienoic acid & 12.71 & - & $\mathrm{C}_{18} \mathrm{H}_{32} \mathrm{O}_{5}$ & 2.7 & 5.1 & 327.2168 & $\begin{array}{c}327.2168(100), 211.1334(39), 229.1439(32), \\
171.1021(19), 239.1284(8)\end{array}$ & - \\
\hline 56. & Unknown & 12.79 & 215 & $\mathrm{C}_{30} \mathrm{H}_{22} \mathrm{O}_{9}$ & 3.3 & 7.3 & 525.1174 & $\begin{array}{c}267.0312(100), 399.0868(95), 361.0711(70), \\
307.0603(40), 349.0712(39)\end{array}$ & - \\
\hline 57. & Unknown & 12.97 & - & $\mathrm{C}_{17} \mathrm{H}_{16} \mathrm{O}_{6}$ & 2.2 & 3.9 & 315.0867 & $\begin{array}{c}315.0867(100), 178.9981(23), 297.0761(20), \\
194.0216(15), 152.0111(11)\end{array}$ & - \\
\hline 58. & Gloriosaol C & 13.14 & 207,327 & $\mathrm{C}_{45} \mathrm{H}_{30} \mathrm{O}_{15}$ & 3.0 & 2.2 & 809.1488 & $\begin{array}{c}267.0295(100), 239.0344(42), 211.0396(8), \\
541.1121(4), 513.1173(3)\end{array}$ & [13] \\
\hline 59. & Unknown & 13.28 & - & $\mathrm{C}_{18} \mathrm{H}_{20} \mathrm{O}_{6}$ & 1.6 & 16.8 & 331.1182 & $285.1127(100)$ & - \\
\hline 60. & Gloriosaol isomer III & 13.63 & - & $\mathrm{C}_{45} \mathrm{H}_{30} \mathrm{O}_{15}$ & 3.2 & 38.4 & 809.1486 & $\begin{array}{c}267.0301(100), 239.0349(36), 508.0790(12), \\
365.0655(11), 463.0835(10)\end{array}$ & - \\
\hline 61. & Trihydroxyoctadecenoic acid & 13.82 & - & $\mathrm{C}_{18} \mathrm{H}_{34} \mathrm{O}_{5}$ & 2.1 & 7.1 & 329.2327 & $\begin{array}{c}329.2326(100), 211.1334(35), 229.1440(26), \\
171.1022(13), 139.1126(3)\end{array}$ & - \\
\hline 62. & Unknown & 14.14 & - & $\mathrm{C}_{45} \mathrm{H}_{30} \mathrm{O}_{14}$ & 2.3 & 28.1 & 793.1545 & $\begin{array}{c}269.0452(100), 267.0297(99), 399.0872(68), \\
125.0239(37), 639.1304(19)\end{array}$ & - \\
\hline 63. & Unknown & 14.14 & - & $\mathrm{C}_{30} \mathrm{H}_{18} \mathrm{O}_{11}$ & 2.0 & 21.9 & 553.0765 & $\begin{array}{c}375.0508(100), 267.0304(74), 525.0813(69), \\
509.0871(60), 457.0916(59)\end{array}$ & - \\
\hline 64. & Unknown & 14.80 & - & $\mathrm{C}_{17} \mathrm{H}_{16} \mathrm{O}_{4}$ & 1.4 & 3.5 & 283.0972 & $\begin{array}{c}283.0972(100), 162.0320(16), 134.0372(2), \\
268.0743(2)\end{array}$ & - \\
\hline 65. & Unknown & 14.89 & - & $\mathrm{C}_{15} \mathrm{H}_{12} \mathrm{O}_{4}$ & 1.4 & 1.6 & 255.0659 & 255.0659 (100) & - \\
\hline 66. & Unknown & 16.68 & - & $\mathrm{C}_{17} \mathrm{H}_{16} \mathrm{O}_{5}$ & 2.0 & 2.4 & 299.0919 & 299.0919 (100), 178.0267 (18), 150.0320 (2) & - \\
\hline 67. & Unknown & 18.82 & - & $\mathrm{C}_{34} \mathrm{H}_{52} \mathrm{O}_{11}$ & 3.2 & 47.2 & 635.3416 & $101.0241(100), 589.3345(11)$ & - \\
\hline 68. & Unknown & 18.93 & - & $\mathrm{C}_{18} \mathrm{H}_{28} \mathrm{O}_{4}$ & 2.0 & 24.8 & 307.1909 & $\begin{array}{c}223.1334(100), 137.0970(63), 265.1806(30), \\
307.1913(20), 185.1183(17)\end{array}$ & - \\
\hline 69. & Unknown & 19.59 & - & $\mathrm{C}_{34} \mathrm{H}_{54} \mathrm{O}_{11}$ & 4.4 & 10.3 & 637.3565 & $161.0450(100), 113.0244(68), 591.3534(16)$ & - \\
\hline 70. & Unknown & 23.78 & - & $\mathrm{C}_{34} \mathrm{H}_{56} \mathrm{O}_{10}$ & 3.2 & 46.4 & 623.3781 & $577.3724(100), 159.0298(63)$ & - \\
\hline 71. & Unknown & 26.68 & - & $\mathrm{C}_{28} \mathrm{H}_{42} \mathrm{O}_{6}$ & 3.3 & 7.0 & 473.2893 & $\begin{array}{c}175.0395(100), 473.2893(23), 297.2427(13), \\
160.0168(11), 193.0499(10)\end{array}$ & - \\
\hline
\end{tabular}

* Accuracy of mass measurements expressed in parts per million (ppm). ${ }^{* *}$ Isotopic pattern fit factor $(\mathrm{m} \sigma)$. 


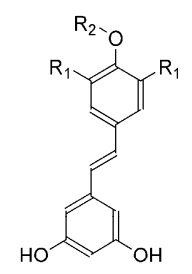
(13): $\mathrm{R}_{1}=\mathrm{OH}, \mathrm{R}_{2}=\mathrm{CH}_{3}$
(21): $\mathrm{R}_{1}=\mathrm{R}_{2}=\mathrm{H}$

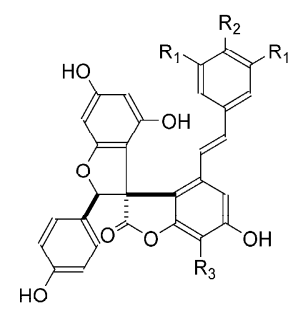

(40): $\mathrm{R}_{1}=\mathrm{OH}, \mathrm{R}_{2}=\mathrm{H}, \mathrm{R}_{3}=\mathrm{OCH}_{3}$ (42): $\mathrm{R}_{1}=\mathrm{OH}, \mathrm{R}_{2}=\mathrm{OCH}_{3}, \mathrm{R}_{3}=\mathrm{H}$ (47): $R_{1}=R_{3}=H, R_{2}=O H$

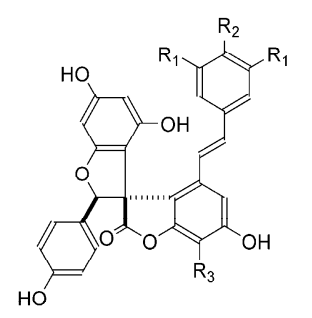

(37): $\mathrm{R}_{1}=\mathrm{OH}, \mathrm{R}_{2}=\mathrm{H}, \mathrm{R}_{3}=\mathrm{OCH}_{3}$ (39): $R_{1}=O H, R_{2}=O_{3}, R_{3}=H$ (48): $R_{1}=R_{3}=H, R_{2}=O H$
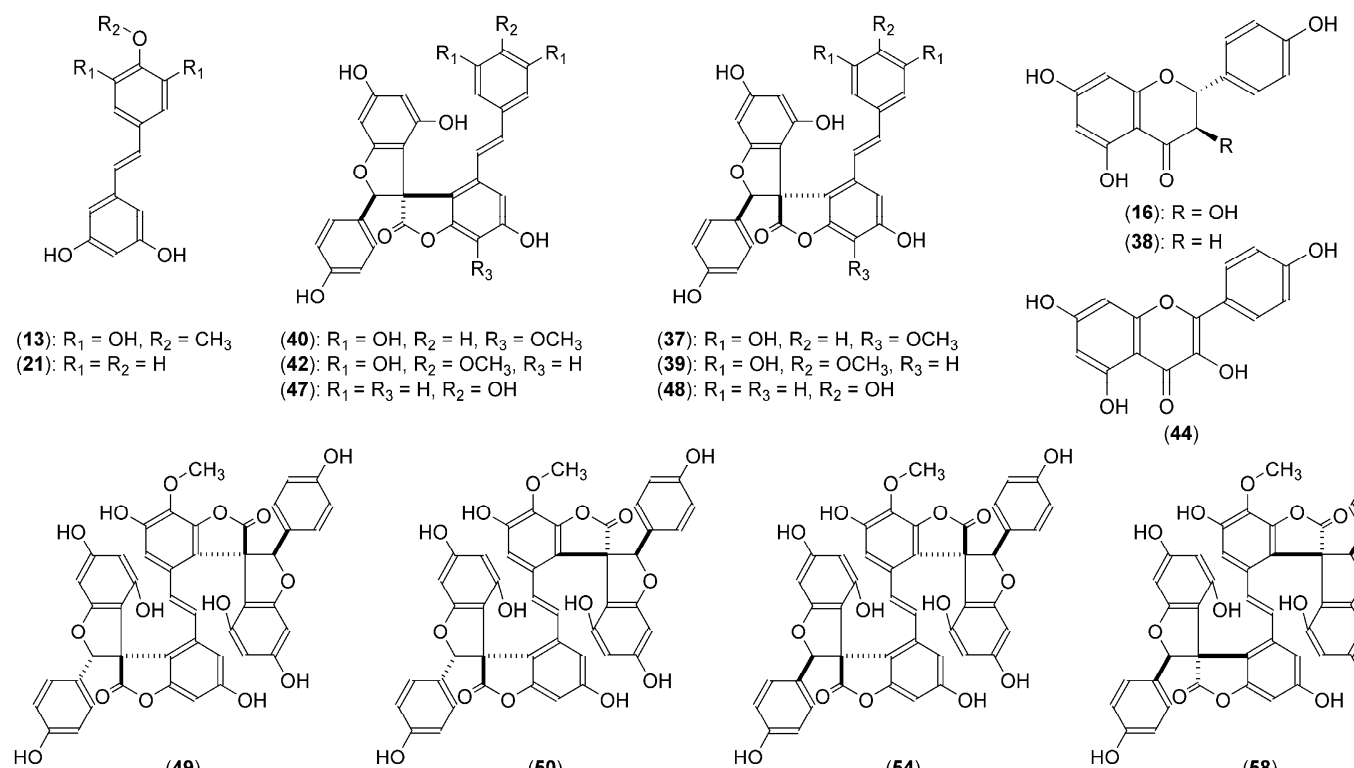

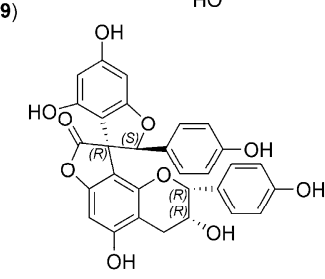

(15)

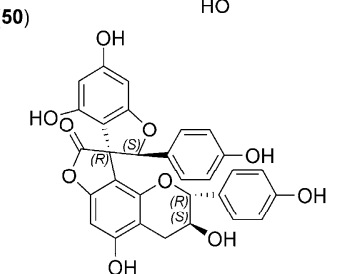

(26)

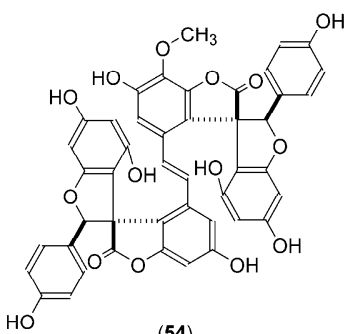

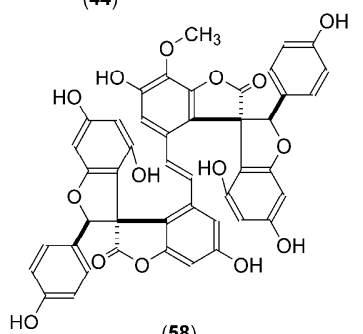

(58)

Figure 2. General structures of phenolic compounds isolated from Y. schidigera bark.

\subsection{Structural Characterization of the New Phenolic Compounds}

Yuccalechin A (15) was isolated as an off-white, amorphous solid exhibiting a UV absorption maximum at $215 \mathrm{~nm}$, with a specific rotation of $[\alpha]^{\mathrm{D}}{ }_{20}=+95$. The negative HRESIMS spectra of 15 showed deprotonated molecule at $\mathrm{m} / \mathrm{z} 541.1131$, and its molecular formula was determined as $\mathrm{C}_{30} \mathrm{H}_{21} \mathrm{O}_{10}$ (calcd. 541.1140). The ${ }^{13} \mathrm{C}-\mathrm{NMR}$ spectra of $\mathbf{1 5}$ showed 25 signals, sorted by the distortionless enhancement by polarization transfer with retention of quaternaries (DEPTQ) and heteronuclear single quantum coherence (HSQC) experiments into $1 \mathrm{CH}_{2}, 9 \mathrm{CH}$, and 15 quaternary carbons with a characteristic signal at $\delta_{C} 177.1$, assignable to the $\gamma$-lactone carbonyl in C-1". The difference in the observed number of carbon atoms compared to HRESIMS was explained by the presence of two similar sets of aromatic protons, corresponding to the para-substituted aromatic groups at $\delta_{\mathrm{H}} 7.15(2 \mathrm{H}, \mathrm{d}$, $\left.J=8.5 \mathrm{~Hz}, \mathrm{H}-2^{\prime} / \mathrm{H}-6^{\prime}\right)$ and $\delta_{\mathrm{H}} 6.72\left(2 \mathrm{H}, \mathrm{d}, J=8.5 \mathrm{~Hz}, \mathrm{H}-3^{\prime} / \mathrm{H}-5^{\prime}\right)$, and at $\delta_{\mathrm{H}} 7.06(2 \mathrm{H}, \mathrm{d}, J=8.5 \mathrm{~Hz}$, $\left.\mathrm{H}-11^{\prime \prime} / \mathrm{H}-15^{\prime \prime}\right)$ and $\delta_{\mathrm{H}} 6.68\left(2 \mathrm{H}, \mathrm{d}, J=8.5 \mathrm{~Hz}, \mathrm{H}-12^{\prime \prime} / \mathrm{H}-14^{\prime \prime}\right)$, due to $\mathrm{AA}^{\prime} \mathrm{XX}^{\prime}$ systems (Table 2). The third aromatic set of meta-coupled protons appeared at $\delta_{\mathrm{H}} 5.81\left(1 \mathrm{H}, \mathrm{d}, J=2.0 \mathrm{~Hz}, \mathrm{H}-6^{\prime \prime}\right)$ and at $\delta_{\mathrm{H}} 5.98(1 \mathrm{H}$, $\left.\mathrm{d}, J=2.0 \mathrm{~Hz}, \mathrm{H}-8^{\prime \prime}\right)$ and suggested the presence of a 2,4,6-tri-O-substituted phenyl group. Additionally, the ${ }^{1} \mathrm{H}-\mathrm{NMR}$ and 2D-COSY (correlation spectroscopy) spectra exhibited three oxygenated methines at $\delta_{\mathrm{H}} 6.25\left(1 \mathrm{H}, \mathrm{s}, \mathrm{H}-3^{\prime \prime}\right)$, correlating in the HSQC spectrum with the spiro-center C-atom at $\delta_{\mathrm{C}} 91.2\left(\mathrm{C}-3^{\prime \prime}\right)$, $\delta_{\mathrm{H}} 4.91(1 \mathrm{H}, \mathrm{br} \mathrm{s}, \mathrm{H}-2)$, and $\delta_{\mathrm{H}} 4.46(1 \mathrm{H}, \mathrm{ddd}, J=4.4,2.9,1.6 \mathrm{~Hz}, \mathrm{H}-3)$, and a methylene group at $\delta_{\mathrm{H}} 2.96$ $(1 \mathrm{H}, \mathrm{dd}, J=17.0,4.4 \mathrm{~Hz}, \mathrm{H}-4 \beta) / 2.90(1 \mathrm{H}, \mathrm{dd}, J=17.0,2.9 \mathrm{~Hz}, \mathrm{H}-4 \alpha)$, suggesting the epiafzelechin-type substructure [66] (Figure 2). 
Table 2. ${ }^{1} \mathrm{H}$ and ${ }^{13} \mathrm{C}-\mathrm{NMR}$ data $\left(\mathrm{MeOH}-d_{4}, 500 / 125 \mathrm{MHz}, 30{ }^{\circ} \mathrm{C}\right)$ for compounds 15, 26, and 29.

\begin{tabular}{|c|c|c|c|c|c|c|c|}
\hline \multirow{2}{*}{ Position } & \multirow{2}{*}{ Type } & \multicolumn{2}{|l|}{15} & \multicolumn{2}{|l|}{26} & \multicolumn{2}{|l|}{29} \\
\hline & & $\begin{array}{c}\delta_{\mathrm{H}} \\
(J, \mathrm{~Hz})\end{array}$ & $\begin{array}{c}\delta_{C} \\
(\mathrm{ppm})\end{array}$ & $\begin{array}{c}\delta_{\mathrm{H}} \\
(J, \mathrm{~Hz})\end{array}$ & $\begin{array}{c}\delta_{C} \\
(\text { ppm })\end{array}$ & $\begin{array}{c}\delta_{\mathrm{H}} \\
(J, \mathrm{~Hz})\end{array}$ & $\begin{array}{c}\delta_{C} \\
(\mathrm{ppm})\end{array}$ \\
\hline 2 & $\mathrm{CH}$ & $4.91 \mathrm{br} \mathrm{s}$ & 79.4 & $4.99 \mathrm{~d}(5.8)$ & 81.6 & $4.19 \mathrm{~d}(9.5)$ & 82.7 \\
\hline 3 & $\mathrm{CH}$ & $4.46 \mathrm{ddd}(4.4,2.9,1.6)$ & 65.6 & $4.05 \mathrm{dt}(5.8,5.5)$ & 68.1 & $3.85 \mathrm{ddd}(9.8,9.5,5.8)$ & 67.7 \\
\hline $4 \alpha$ & $\mathrm{CH}_{2}$ & $2.90 \mathrm{dd}(17.0,2.9)$ & 29.6 & $2.68 \mathrm{~d}(5.5)$ & 26.8 & $2.39 \mathrm{dd}(16.3,9.8)$ & 30.5 \\
\hline $4 \beta$ & & $2.96 \mathrm{dd}(17.0,4.4)$ & & & & $2.93 \mathrm{dd}(16.3,5.8)$ & \\
\hline 5 & $\mathrm{C}$ & & 158.4 & & 158.0 & & 157.6 \\
\hline 6 & $\mathrm{CH}$ & $6.16 \mathrm{~s}$ & 91.6 & $6.14 \mathrm{~s}$ & 91.5 & $6.05 \mathrm{~s}$ & 91.1 \\
\hline 7 & C & & 153.8 & & 154.1 & & 152.8 \\
\hline 8 & C & & 106.7 & & 105.8 & & 106.1 \\
\hline $4 a$ & $\mathrm{C}$ & & 104.8 & & 104.9 & & 105.6 \\
\hline $8 a$ & C & & 153.1 & & 152.1 & & 153.2 \\
\hline $1^{\prime}$ & $\mathrm{C}$ & & 130.6 & & 131.2 & & 130.1 \\
\hline $2^{\prime}$ & $\mathrm{CH}$ & $7.15 \mathrm{~d}(8.5)$ & 128.7 & $6.98 \mathrm{~d}(8.5)$ & 128.2 & $7.20 \mathrm{~d}(8.5)$ & 130.4 \\
\hline $3^{\prime}$ & $\mathrm{CH}$ & $6.72 \mathrm{~d}(8.5)$ & 115.8 & $6.66 \mathrm{~d}(8.5)$ & 115.9 & $6.83 \mathrm{~d}(8.5)$ & 115.7 \\
\hline $4^{\prime}$ & $\mathrm{C}$ & & 157.7 & & 157.8 & & 158.3 \\
\hline $5^{\prime}$ & $\mathrm{CH}$ & $6.72 \mathrm{~d}(8.5)$ & 115.8 & $6.66 \mathrm{~d}(8.5)$ & 115.9 & $6.83 \mathrm{~d}(8.5)$ & 115.7 \\
\hline $6^{\prime}$ & $\mathrm{CH}$ & $7.15 \mathrm{~d}(8.5)$ & 128.7 & $6.98 \mathrm{~d}(8.5)$ & 128.2 & $7.20 \mathrm{~d}(8.5)$ & 130.4 \\
\hline $1 "$ & $\mathrm{C}$ & & 177.1 & & 177.1 & & 180.6 \\
\hline $2^{\prime \prime}$ & $\mathrm{C}$ & & 61.5 & & 61.5 & & 61.9 \\
\hline 3" & $\mathrm{CH}$ & $6.25 \mathrm{~s}$ & 91.2 & $6.13 \mathrm{~s}$ & 90.6 & $5.72 \mathrm{~s}$ & 94.5 \\
\hline $4 "$ & $\mathrm{C}$ & & 105.7 & & 105.8 & & 106.1 \\
\hline $5^{\prime \prime}$ & $\mathrm{C}$ & & 156.1 & & 156.2 & & 155.1 \\
\hline $6^{\prime \prime}$ & $\mathrm{CH}$ & $5.81 \mathrm{~d}(2.0)$ & 96.7 & $5.91 \mathrm{~d}(2.0)$ & 96.7 & $5.78 \mathrm{~d}(2.0)$ & 96.5 \\
\hline $7^{\prime \prime}$ & C & & 161.3 & & 161.4 & & 161.0 \\
\hline $8^{\prime \prime}$ & $\mathrm{CH}$ & $5.98 \mathrm{~d}(2.0)$ & 90.6 & $5.94 \mathrm{~d}(2.0)$ & 90.8 & $5.66 \mathrm{~d}(2.0)$ & 90.7 \\
\hline $9^{\prime \prime}$ & C & & 164.8 & & 164.6 & & 163.8 \\
\hline $10^{\prime \prime}$ & $\mathrm{C}$ & & 128.3 & & 128.1 & & 128.1 \\
\hline $11^{\prime \prime}$ & $\mathrm{CH}$ & $7.06 \mathrm{~d}(8.5)$ & 128.5 & $7.03 \mathrm{~d}(8.5)$ & 128.4 & $7.07 \mathrm{~d}(8.5)$ & 128.4 \\
\hline $12 "$ & $\mathrm{CH}$ & $6.68 \mathrm{~d}(8.5)$ & 115.8 & $6.68 \mathrm{~d}(8.5)$ & 115.8 & $6.60 \mathrm{~d}(8.5)$ & 115.5 \\
\hline $13^{\prime \prime}$ & $\mathrm{C}$ & & 158.6 & & 158.6 & & 158.3 \\
\hline $14 "$ & $\mathrm{CH}$ & $6.68 \mathrm{~d}(8.5)$ & 115.8 & $6.68 \mathrm{~d}(8.5)$ & 115.8 & $6.60 \mathrm{~d}(8.5)$ & 115.5 \\
\hline $15^{\prime \prime}$ & $\mathrm{CH}$ & $7.06 \mathrm{~d}(8.5)$ & 128.5 & $7.03 \mathrm{~d}(8.5)$ & 128.4 & $7.07 \mathrm{~d}(8.5)$ & 128.4 \\
\hline
\end{tabular}

Analysis of long-range correlations visible in the heteronuclear multiple bond coherence (HMBC) spectrum gave characteristic cross-peaks (Figure 3) between H-3" and spiro-center C-2" ( $\delta 61.5)$, $\gamma$-lactone C-1" ( $\delta$ 177.1), p-hydroxyphenyl C-10" ( $\delta 128.3)$, and dihydrobenzopyran C-8 ( $\delta$ 106.7), while the H-2 correlated with C-8a $(\delta 153.1)$ and C-1' $(\delta 130.6)$ of the B-ring. These data were in close agreement with those of larixinol [16,67].

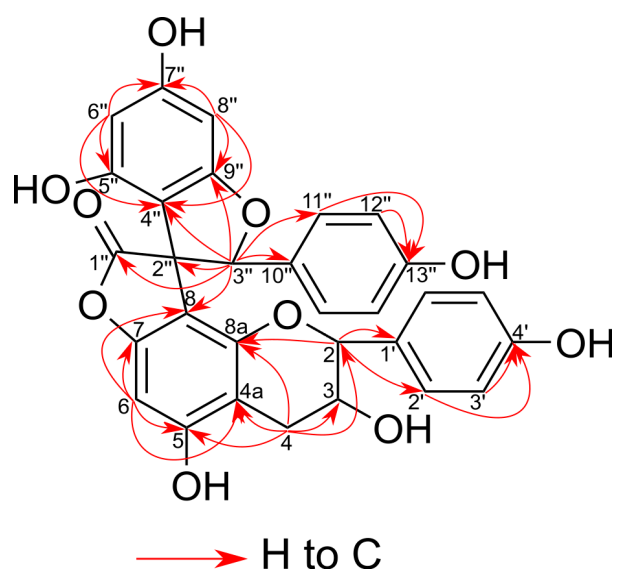

Figure 3. Key heteronuclear multiple bond coherence (HMBC) correlations for compounds 15, 26, and 29.

The flavan-3-ol compounds, like (+)-(2R,3S)-catechin and (-)-(2R,3R)-epicatechin, consist of two benzene rings (A and $\mathrm{B}$ ) and a pyran $\mathrm{C}$-ring. They have two stereocenters and, therefore, four possible diastereomers, 2,3-trans- $(2 R, 3 S) /(2 S, 3 R)$ and $2,3-$ cis- $(2 R, 3 R) /(2 S, 3 S)$, with the C-ring being conformationally flexible. The rapid flexing within the C-ring can bring the B-ring into a 
pseudoequatorial (E-conformer) or a pseudoaxial (A-conformer) position (Figure 4). The equilibrium between different forms depends on the solvent used, hence $\mathrm{H}-\mathrm{H}$ and $\mathrm{C}-\mathrm{H}$ coupling constants should be considered as time-averaged values (e.g., A:E ratios of about 41:59 (DMSO), 30:70 (dioxane), and 33:67 (water) for (+)-catechin) [68,69]. The 2,3-trans-flavan-3-ols showed the preference of the B-ring to be in an equatorial position, while for the 2,3-cis-compounds the distorted equatorial position, or a significant population of the axial C-2 aryl conformations was present [70].

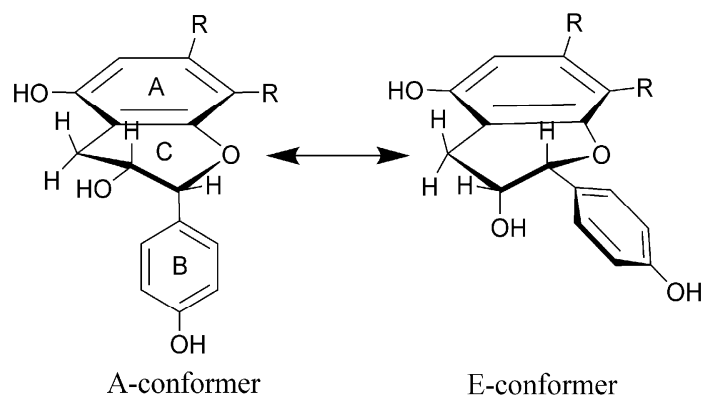

Figure 4. Structures of A and E conformers. Ring B is in a pseudo-axial position in the A-conformer and in a pseudo-equatorial position in the E-conformer.

According to the small coupling constant $(J \approx 2 \mathrm{~Hz})$, protons $\mathrm{H}-2$ and $\mathrm{H}-3$ in $\mathbf{1 5}$ were deduced as having cis-relative configurations, similarly to epicatechin [71] and epiafzelechin [66]. The orientation of protons $\mathrm{H}-2$ and $\mathrm{H}-3$ can be deduced also from the direct $\mathrm{H}-\mathrm{C}$ coupling constants, which depend on the stereochemistry of the heteroatom-substituted cyclohexane C-ring, presenting smaller values for axial $\mathrm{H}-\mathrm{C}$ than for the equatorial atoms (typically $\Delta J=4 \mathrm{~Hz}$ ) - the so-called normal Perlin effect-which reflects the greater length of the axial $\mathrm{C}$-H bonds. The bond lengthening in the axial position occurs as a result of hyper-conjugative $\sigma_{\mathrm{C}-\mathrm{H}} \rightarrow \sigma^{*} \mathrm{C}-\mathrm{H}$ interactions of anti-periplanar $\mathrm{C}-\mathrm{H}$ bonds in the axial position [72,73]. On the other hand, one has to remember that axial protons at the $\beta$-carbons (i.e., $C$-3 of C-ring) of hetero-substituted cyclohexanes could also present coupling constants larger than those of the equatorial protons (the reverse Perlin effect) [74,75], but no evidence was found to substantiate it in the case of 15. Furthermore, the ${ }^{1} J_{\mathrm{CH}}$ coupling displays an angular dependence for $\mathrm{C}-\mathrm{H}$ bonds adjacent to $\pi$-bonds. In many cases, $\sigma_{\mathrm{C}-\mathrm{H}} \rightarrow \pi^{*}$ interactions lengthen $\mathrm{C}-\mathrm{H}$ bonds, with maximized overlap of interacting orbitals when the $\mathrm{C}-\mathrm{H}$ bond is aligned with the $\pi$-bond, thus giving the minimum ${ }^{1} J_{\mathrm{CH}}$ value [76]. In order to explore these previously undescribed properties of flavan-3-ols, we measured a series of $\mathrm{C}-\mathrm{H}$ couplings $\left({ }^{1} \mathrm{~J}_{\mathrm{CH}},{ }^{2} J_{\mathrm{CH}}\right.$ and $\left.{ }^{3} \mathrm{~J}_{\mathrm{CH}}\right)$ using the F2-coupled HSQC [77] and HSQC-HECADE experiments [78] for (+)-catechin and (-)-epicatechin (close analogs of (+)-afzelechin and (-)-epiafzelechin) and compounds 15, 26, and 29 (Table 3). The accurate values of ${ }^{1} J_{\mathrm{CH}}$ for $\mathrm{C}-2$ $(144.0 \mathrm{~Hz})$ and C-3 $(146.2 \mathrm{~Hz})$ for compound 15 suggested that H-2 adopted a pseudoaxial, and H-3 a pseudoequatorial orientation.

Table 3. Experimental ${ }^{1} J_{\mathrm{CH}},{ }^{2} J_{\mathrm{CH}}$, and ${ }^{3} J_{\mathrm{CH}}(\mathrm{J}$ in Hz) couplings of $\mathrm{C}-2$ and $\mathrm{C}-3$ for $(+)$-catechin $(R, S)$, $(-)$-epicatechin $(R, R)$, and compounds 15, 26, and 29.

\begin{tabular}{lccccc}
\hline$J$-Type & (+)-Catechin & (-)-Epicatechin & $\mathbf{1 5}$ & $\mathbf{2 6}$ & $\mathbf{2 9}$ \\
\hline${ }^{1} J_{\mathrm{C} 2-\mathrm{H} 2}$ & 146.1 & 142.9 & 144.0 & 148.9 & 144.7 \\
${ }^{1} J_{\mathrm{C} 3-\mathrm{H} 3}$ & 145.3 & 146.2 & 146.2 & 146.0 & 144.8 \\
${ }^{2} J_{\mathrm{C} 3-\mathrm{H} 2}$ & -4.0 & +1.0 & +1.4 & -4.7 & -2.5 \\
${ }^{2} J_{\mathrm{C} 2-\mathrm{H} 3}$ & -1.6 & +1.9 & +1.5 & 0.0 & -3.3 \\
${ }^{2} J_{\mathrm{C} 4-\mathrm{H} 3}$ & -1.0 & +2.0 & -2.2 & -1.0 & -0.9 \\
${ }^{3} J_{\mathrm{C} 4-\mathrm{H} 2}$ & +3.2 & +1.8 & +2.1 & +3.5 & +3.0 \\
\hline
\end{tabular}

Additionally, ${ }^{2} \mathrm{~J}_{\mathrm{CH}}$ coupling provides conformational information that can identify the position of an oxygen functionality-when it is gauche to its geminal proton, the coupling becomes large 
$(4-7 \mathrm{~Hz})$, and when it is anti, ${ }^{2} J_{\mathrm{CH}}$ becomes small $(0-2 \mathrm{~Hz})$. On the other hand, ${ }^{3} J_{\mathrm{CH}}$ coupling follows a Karplus-type dependence, thus showing smaller values for gauche $(1-3 \mathrm{~Hz})$ than for anti-periplanar conformation $(5-8 \mathrm{~Hz})[79,80]$. The data gathered in Table 3 for compound 15 agreed with the relative 2,3-cis-configuration.

The relative configuration between the C-2"/C-3" of the benzofuran F-ring was deduced primarily on the basis of the chemical shift of C-1"(177.1), as reported by Nakashima et al. [15]. The article reported that ${ }^{13} \mathrm{C}$ NMR spectra of yuccaols A-E and gloriosaols $\mathrm{A}-\mathrm{E}$ in $\mathrm{CD}_{3} \mathrm{OD}$ showed the $\gamma$-lactone carbonyl atoms appearing within range $\delta_{C}$ 174.6-176.9 for compounds with cis-configurations between C-2" and C-3", while for those with trans-configuration between $\delta_{C}$ 178.6-181.1. Interestingly, we observed that yuccaols A-E, yuccalide A and gloriosaol A, and gloriosaols C-E isolated in this study had ${ }^{1} J_{\mathrm{CH}}$ couplings measured for the C-3" appearing within range 154.2-155.0 Hz for compounds with the cis-configuration between $C-2$ " and $C-3$ " (2" $R, 3$ " $S$ or 2 " $S, 3$ " $R$ ), and 152.9-153.3 Hz for compounds adopting the trans-configuration $\left(2 " R, 3\right.$ " $R$ or $2^{\prime \prime} S, 3$ " $\left.S\right)$, and they were inversely correlated with the chemical shifts of corresponding C-1" carbon atoms. The ${ }^{1} J_{\mathrm{CH}}$ for C-3" in compounds 15, 26, and 29 were 157.2 (cis-), 156.3 (cis-), and $150.8 \mathrm{~Hz}$ (trans-), respectively, while the chemical shifts of C-1" were $177.1,177.1$, and 180.6, respectively. Additionally, the DP4+ probability calculation was implemented as confirmation for establishing the relative configuration of $\mathbf{1 5}$. Based on the aforementioned observation and 2D-ROESY correlations from H-3" to $\mathrm{H}-2$ and to $\mathrm{H}-2^{\prime} / \mathrm{H}-6^{\prime}$ (Figure 5), the relative configuration of 15 was determined as $2^{\prime \prime} R^{*}, 3^{\prime \prime} S^{*}, 2 R^{*}, 3 R^{*}$.

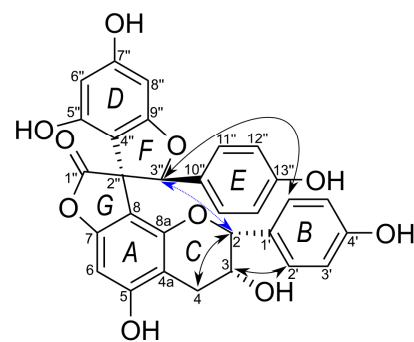

(15)

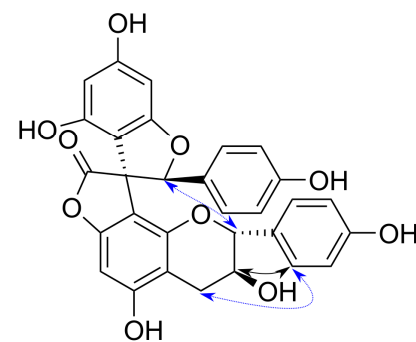

(26)

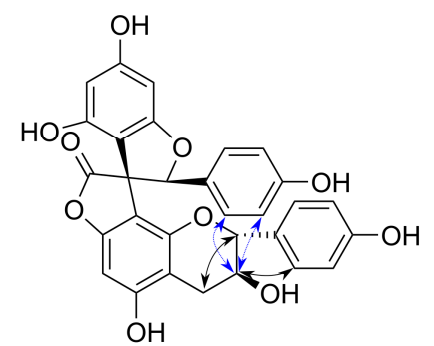

(29)

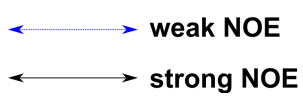

Figure 5. Structures of compounds 15, 26, and 29 isolated from the bark of $Y$. schidigera and key nuclear Overhauser effect (NOE) correlations.

However, because of the flexibility of the C-ring, to confirm the stereochemistry of chiral centers $\mathrm{C}-2$ and $\mathrm{C}-3$, all the possible isomers had to be generated and optimized using the DFT/B3LYP/6-31G(d,p)/IEFPCM/methanol (B3LYP = Becke, 3-parameter, Lee-Yang-Parr; IEFPCM $=$ integral equation formalism for the polarizable continuum model) level of theory. Among them, only $2^{\prime \prime} R^{*}, 3^{\prime \prime} S^{*}, 2 R^{*}, 3 R^{*}$ and $2^{\prime \prime} R^{*}, 3^{\prime \prime} S^{*}, 2 S^{*}, 3 R^{*}$ were consistent with observed NOEs and coupling constants. Subsequently, geometrical optimization and further NMR calculations of the two isomers were performed using the gauge-independent atomic orbital (GIAO) method by implementing mpw1pw91/6-311G+ (d,p)/IEFPCM/methanol. The DP4+ probability calculation indicated that the probability of all atoms (sum of scaled and unscaled probabilities of all $\mathrm{H}$ and $\mathrm{C}$ atoms) for isomer $2^{\prime \prime} R^{*}, 3^{\prime \prime} S^{*}, 2 R^{*}, 3 R^{*}$ was $100 \%$, which was in accordance with the observed coupling constants (Figure S11). Furthermore, the mean average error (MAE) and corrected mean average error (CMAE) values along with correlation coefficients were calculated to evaluate correct/incorrect assignments. As shown in Table 4, MAE and CMAE values showed higher errors for incorrect isomers, confirming the relative configuration of 15 as for $2^{\prime \prime} R^{*}, 3^{\prime \prime} S^{*}, 2 R^{*}, 3 R^{*}$. 
Table 4. Calculated mean average error (MAE), corrected MAE (CMAE), and correlation coefficients of the pair of possible diastereomers for each compound.

\begin{tabular}{|c|c|c|c|c|c|c|}
\hline \multirow{2}{*}{ Parameters } & \multicolumn{2}{|c|}{15} & \multicolumn{2}{|c|}{26} & \multicolumn{2}{|c|}{29} \\
\hline & $2^{\prime \prime} R, 3^{\prime \prime} S, 2 S, 3 R$ & $2^{\prime \prime} R, 3^{\prime \prime} S, 2 R, 3 R$ & $2^{\prime \prime} R, 3^{\prime \prime} S, 2 R, 3 S$ & $2^{\prime \prime} R, 3^{\prime \prime} S, 2 S, 3 S$ & $2^{\prime \prime} R, 3^{\prime \prime} R, 2 S, 3 R$ & $2^{\prime \prime} R, 3^{\prime \prime} R, 2 R, 3 S$ \\
\hline${ }^{1} \mathrm{H}-\mathrm{MAE}$ & 3.5 & 3.40 & 3.8 & 3.53 & 0.38 & 0.4 \\
\hline${ }^{13} \mathrm{C}-\mathrm{MAE}$ & 5.6 & 5.40 & 5.5 & 5.18 & 5.23 & 4.95 \\
\hline Total MAE & 4.85 & 4.44 & 4.92 & 4.60 & 3.54 & 3.37 \\
\hline${ }^{1} \mathrm{H}-\mathrm{CMAE}$ & 4.16 & 3.69 & 3.29 & 3.9 & 0.83 & 0.7 \\
\hline${ }^{13} \mathrm{C}-\mathrm{CMAE}$ & 10.92 & 10.74 & 9.53 & 10.45 & 10.65 & 9.85 \\
\hline Total CMAE & 8.57 & 8.03 & 8.40 & 8.17 & 7.23 & 6.67 \\
\hline$r \mathrm{H}$ & 0.9703 & 0.9724 & 0.9772 & 0.9826 & 0.98460 & 0.93860 \\
\hline$r \mathrm{C}$ & 0.9966 & 0.9977 & 0.9983 & 0.9986 & 0.99900 & 0.99770 \\
\hline Total $r$ & 0.98995 & 0.99203 & 0.99377 & 0.99506 & 0.99608 & 0.98812 \\
\hline
\end{tabular}

In order to determine the absolute configuration of 15 , the 3D structure was optimized by conformational analysis (refer to method section). All conformers occurring in the energy window of $5 \mathrm{kcal} \mathrm{mol}^{-1}$ were subjected to geometrical optimization and minimization (Figure S10). Further calculations of excitation states, rotatory strength, and, hence, simulated ECD spectrum were performed by the TD-DFT/B3LYP/6-31G(d,p)/IEFPCM/methanol level of theory. All spectra obtained were Boltzmann averaged and, after applying UV correction of $+10 \mathrm{~nm}$ and half band of $0.2 \mathrm{eV}$, compared to the experimentally obtained spectrum of 15 , recorded in methanol. The ECD spectrum calculated was in good agreement with the experimental one (Figure 6), while its enantiomer showed opposite Cotton effects. Therefore, the absolute configuration of $\mathbf{1 5}$ was assigned as $2^{\prime \prime} R, 33^{\prime \prime} S, 2 R, 3 R$.

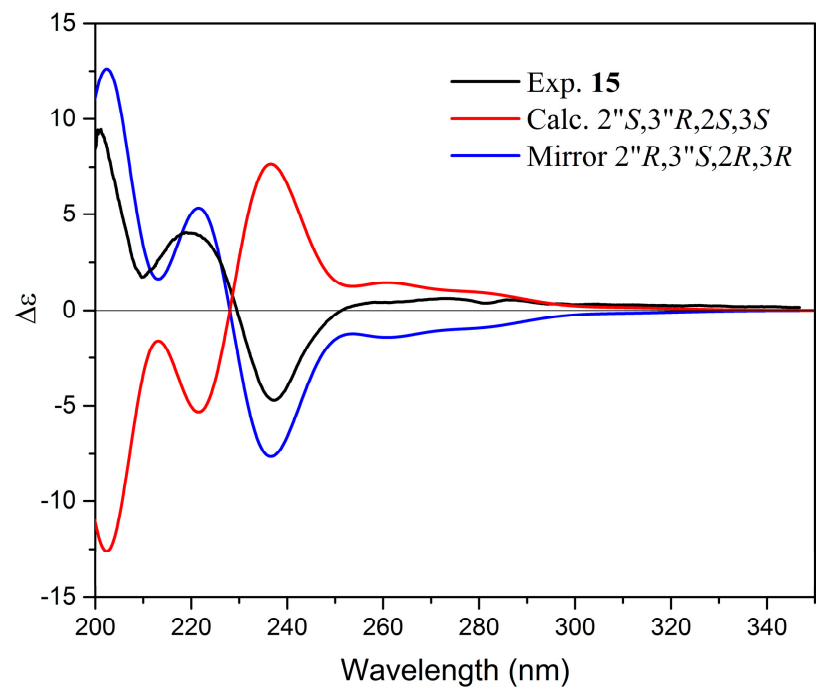

Figure 6. Comparison of experimental and calculated electronic circular dichroism (ECD) spectra of 15.

Finally, the structure of yuccalechin A, a new spirobiflavonoid, was established as $\left(2 S, 2^{\prime} R, 3 R, 3^{\prime} R\right)$ $3^{\prime}, 4,5^{\prime}, 6$-tetrahydroxy-2,2' -bis(4-hydroxyphenyl)-3' , $4^{\prime}$-dihydro- $2 H, 2^{\prime} H, 8^{\prime} H$-spiro[benzofuran-3, $9^{\prime}$-furo [2,3-h]chromen]-8'-one.

Yuccalechin B (26) was obtained as an off-white, amorphous solid exhibiting UV absorption maximum at $210 \mathrm{~nm}$, with a specific rotation of $[\alpha]^{\mathrm{D}_{20}}=-175$. The negative HRESIMS spectra of $\mathbf{2 6}$ showed a deprotonated molecule at $\mathrm{m} / \mathrm{z} 541.1123$, and its molecular formula was determined as $\mathrm{C}_{30} \mathrm{H}_{21} \mathrm{O}_{10}$ (calcd 541.1140), the same as $\mathbf{1 5}$. The ${ }^{1} \mathrm{H}$ and ${ }^{13} \mathrm{C}-\mathrm{NMR}$ spectra of compound 26 were similar to those of 15, except for the signals of H-2 $(\delta 4.99,1 \mathrm{H}, \mathrm{d}, J=5.8 \mathrm{~Hz}) / \mathrm{C}-2(\delta 81.6), \mathrm{H}-3(\delta 4.05$, $1 \mathrm{H}, \mathrm{dt}, J=5.8,5.5 \mathrm{~Hz}) / \mathrm{C}-3(\delta 68.1), \mathrm{H}-4(\delta 2.68,2 \mathrm{H}, \mathrm{d}, J=5.5 \mathrm{~Hz}) / \mathrm{C}-4(\delta 26.8), \mathrm{H}-2^{\prime} / \mathrm{H}-6^{\prime}(\delta 6.98,2 \mathrm{H}, \mathrm{d}$, $J=8.5 \mathrm{~Hz})$, and of H-3" $(\delta 6.13,1 \mathrm{H}, \mathrm{s})$ (Table 2). The analysis of 2D-NMR spectra provided sufficient data to ascribe the planar structure of $\mathbf{2 6}$ as identical with the one of 15, but the difference in the observed retention time (Figure 1, Table 1) and different optical rotation suggested that compound 26 
was a stereoisomer of 15 . The ${ }^{1} \mathrm{H}-{ }^{1} \mathrm{H}$ vicinal coupling constants observed for the $\mathrm{C}$-ring suggested that it adopted 2,3-trans-configuration (afzelechin-type), with a di-pseudo-equatorial orientation of $\mathrm{H}-2 / \mathrm{H}-3$, as suggested by the large ${ }^{1} J_{\mathrm{CH}}$ coupling constants, large ${ }^{2} J_{\mathrm{C} 3-\mathrm{H} 2}$, and small ${ }^{2} J_{\mathrm{C} 2-\mathrm{H} 3}$, which were reasonably similar to that of (+)-catechin (Table 3 ) and NOEs observed between $\mathrm{H}-2^{\prime} / \mathrm{H}-6^{\prime}$ and both H-3 and H-4 (Figure 5). The relative configuration between C-2"/C-3" of the benzofuran ring was deduced from the chemical shift of C-1" (177.1) and ${ }^{1} \mathrm{~J}_{\mathrm{CH}}$ for C-3" $(156.3 \mathrm{~Hz})$; therefore, it was a cis-isomer. In order to establish and prove the relative configuration of $\mathrm{C}-2 / \mathrm{C}-3$, possible isomers of the C-ring-2" $R, 3$ " $S, 2 R, 3 S, 2$ " $R, 3$ " $S, 2 S, 3 R$, and $2 " S, 3$ " $S, 2 S, 3 S$-were generated and imported to conformational analysis followed by geometrical optimization (Figure S24) and DP4+ probability analysis of calculated chemical shifts. However, after the first optimization step, isomer 2 " $R, 3$ " $S, 2 S, 3 R$ was excluded from further calculation. There were two reasons for this: a) the lowest energy and most stable conformers showed over space correlations between $\mathrm{H}-3^{\prime \prime}$ and $\mathrm{H}-2^{\prime} / 6^{\prime}$, while this is the connection only observed in the case of compound 15, and b) low-energy conformers of 2 " $R, 3$ " $S, 2 S, 3 R$ showed ax-eq configuration between $\mathrm{H}-2$ and $\mathrm{H}-3$, which were not in agreement with observed coupling constants. The DP4+ calculations showed that $2 " R, 3$ " $S, 2 R, 3 S$ with a probability of $99.19 \%$ was the correct isomer (Figure S25). The calculated MAE and CMAE values were lower for the correct isomer and further confirmed by the higher correlation coefficient value for that one. Overall, the results obtained were compatible with the result of DP4+ probability calculations (Table 4). Therefore, the relative configuration of $\mathbf{2 6}$ was established as $2^{\prime \prime} R^{*}, 3^{\prime \prime} S^{*}, 2 R^{*}, 3 S^{*}$. Furthermore, to determine the absolute configuration of 26, optimized conformers were subjected to ECD spectra simulations using TD-DFT/cam-B3LYP/6-31G(d,p)/IEFPCM/methanol. A comparison of the Boltzmann-averaged spectrum with one obtained experimentally, after applying UV correction of $+23 \mathrm{~nm}$ and half band of $0.25 \mathrm{eV}$, resulted in the absolute configuration of $\mathbf{2 6}$ determined as $2^{\prime \prime} R, 33^{\prime \prime} S, 2 R, 3 S$ (Figure 7). This outcome coincided with the structure of 3,2'-epi-larixinol, a compound isolated from the aerial parts of Abies chensiensis [19], but differing in both NMR chemical shifts and optical rotation compared to 26 . However, the authors did not imply the AC of the aforementioned compound and relied solely on NOEs in determining the relative configuration; thus, our findings still hold true.

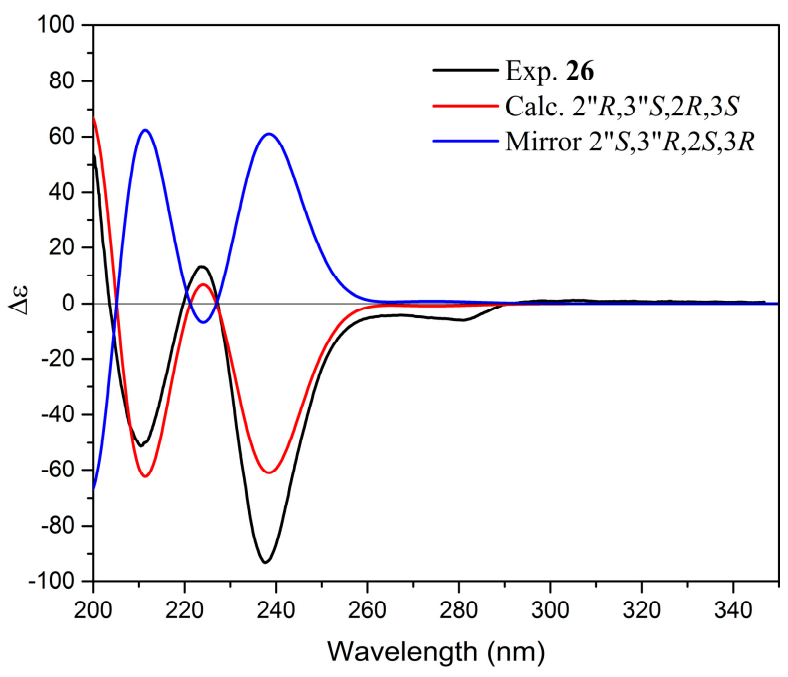

Figure 7. Comparison of experimental and calculated ECD spectra of 26.

In this way, the structure of yuccalechin B was established as $\left(2 S, 2^{\prime} R, 3 R, 3^{\prime} S\right)-3^{\prime}, 4,5^{\prime}, 6$-tetrahydroxy2,2' -bis(4-hydroxyphenyl)-3' , $^{\prime}$-dihydro- $2 H, 2^{\prime} H, 8^{\prime} H$-spiro[benzofuran-3,9'-furo.[2,3- $h$ ]chromen]-8'-one.

Yuccalechin C (29) was obtained as an off-white, amorphous solid exhibiting UV absorption maximum at $211 \mathrm{~nm}$, with a specific rotation of $[\alpha]^{\mathrm{D}_{20}}=+148$. The negative HRESIMS spectra of 29 showed a deprotonated molecule at $\mathrm{m} / \mathrm{z} 541.1121$, and its molecular formula was determined as $\mathrm{C}_{30} \mathrm{H}_{21} \mathrm{O}_{10}$ (calcd 541.1140), the same as $\mathbf{1 5}$ and $\mathbf{2 6}$. The planar structure of $\mathbf{2 9}$ was assigned to 
be identical to compounds $\mathbf{1 5}$ and $\mathbf{2 6}$ based on the analysis of $1 \mathrm{D}$ and 2D-NMR spectra. However, the ${ }^{1} \mathrm{H}$ and ${ }^{13} \mathrm{C}-\mathrm{NMR}$ spectra presented certain differences when compared to yuccalechin $\mathrm{B}(26)$, namely signals of upfield-shifted H-2 $(\delta 4.19,1 \mathrm{H}, \mathrm{d}, J=9.5 \mathrm{~Hz}), \mathrm{H}-3(\delta 3.85,1 \mathrm{H}$, ddd, $J=9.8,9.5$, $5.8 \mathrm{~Hz})$, and $\mathrm{H}-3{ }^{\prime \prime}(\delta 5.72,1 \mathrm{H}, \mathrm{s})$ and downfield-shifted C-3 $(\delta 30.5), \mathrm{C}-1 " \mathrm{l}(\delta 180.6)$, and C-3" ( $\left.\delta 94.5\right)$. These changes, along with a difference in chromatographic behavior ( $\mathrm{RT}=8.93 \mathrm{~min}$ vs. $8.39 \mathrm{~min}$ for 26) and optical rotation, indicated that compound 29 was a stereoisomer of $\mathbf{1 5}$ and 26. Similarly to 26, protons $\mathrm{H}-2$ and $\mathrm{H}-3$ of the C-ring were in 2,3-trans-configuration (afzelechin-type), but with an energetically favorable di-axial orientation, as indicated by a large ${ }^{3} \int_{\mathrm{H}-\mathrm{H}}$ coupling constant, small ${ }^{1} J_{\mathrm{CH}}$ coupling constants, relatively large ${ }^{2} J_{\mathrm{C} 3-\mathrm{H} 2}$ and ${ }^{2} J_{\mathrm{C} 2}-\mathrm{H} 3$ (Table 3 ), and NOEs observed between $\mathrm{H}-2^{\prime} / \mathrm{H}-6^{\prime}$ and $\mathrm{H}-3$ and between $\mathrm{H}-2$ and $\mathrm{H}-4 \beta$ (Figure 5). The relative configuration of the spiro-center was apparent from the chemical shift of C-1" $(\delta 180.6)$ and ${ }^{1} J_{\mathrm{CH}}$ for $\mathrm{C}-3^{\prime \prime}(150.8 \mathrm{~Hz})$, and as a result, the relative configuration between C-2"/C-3" of the F-ring was deduced as trans. Subsequently, to prove the relative configuration, two possible isomers 2 " $R, 3$ " $R, 2 S, 3 R$ and 2 " $R, 3$ " $R, 2 R, 3 S$ were subjected to an NMR calculation followed by a DP4+ probability calculation. Results implied that the $2 " R, 3 " R, 2 S, 3 R$ isomer was the correct isomer with a probability value of $100 \%$ (Figure S35). Although the MAE and CMAE values were lower and in favor of the incorrect isomer, the correlation coefficient was higher for correct assignment $(\Delta r=0.00795)$. Additionally, the ECD calculation of optimized structures (Figure S34) was performed using TD-DFT/cam-B3LYP/6-31G(d,p)/CPCM/methanol (CPCM $=$ conductor-like polarizable continuum model) and resulted in establishing the absolute configuration of 29 as $2^{\prime \prime} S, 3 " S, 2 R, 3 S$ (Figure 8).

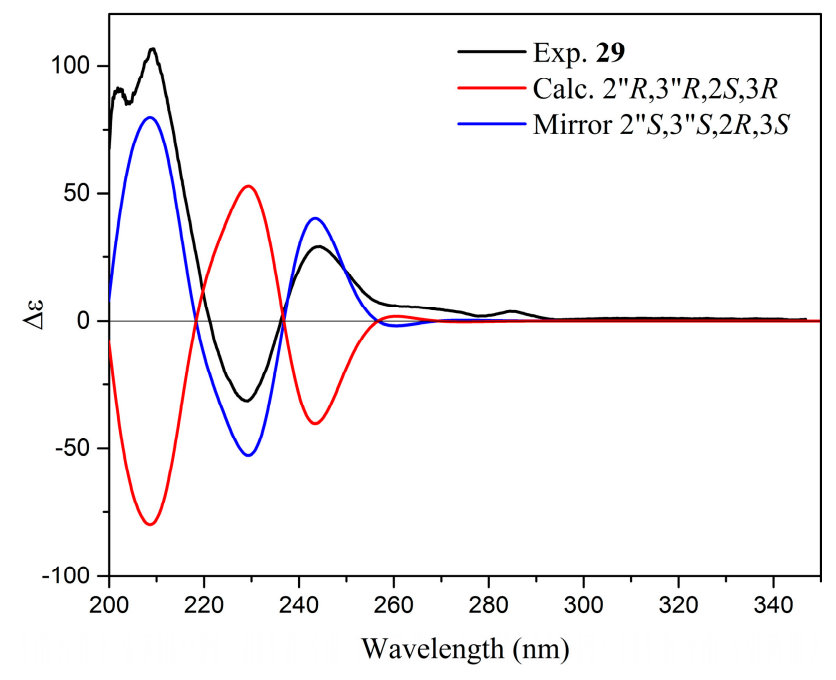

Figure 8. Comparison of experimental and calculated ECD spectra of 29.

As a result, the structure of yuccalechin $C$ was elucidated as $\left(2 S, 2^{\prime} R, 3 S, 3^{\prime} S\right)-3^{\prime}, 4,5^{\prime}, 6$-tetrahydroxy2,2' -bis(4-hydroxyphenyl)-3' , $4^{\prime}$-dihydro-2 $H, 2^{\prime} H, 8^{\prime} H$-spiro[benzofuran-3, $9^{\prime}$-furo[2,3-h]chromen]-8' -one.

2.3. Anti-Cholinesterase Activities of trans-3,3',5,5'-Tetrahydroxy-4'-methoxystilbene and Yuccalechins B and C

The modified Ellman's assay was used to measure the anti-acetylcholinesterase and anti-butyrylcholinesterase activities of 13, 26, and 29 [81,82]. As a result, spirobiflavonoids (26 and 29) had very low and selective activities against AChE, while the stilbene derivative, trans-3,3',5,5'-tetrahydroxy-4'-methoxystilbene (13), was almost inactive against AChE/BChE (Table 5). The result obtained for the latter seems to be in line with our previous results, showing very weak activity of trans-resveratrol and much higher, selective against $\mathrm{BChE}$, activity of piceatannol (trans-3,3', 4', 5-tetrahydroxystilbene) [83]. 
Table 5. Inhibition (\% \pm S.D.) and $\mathrm{IC}_{50}$ values of compounds 13, 26, and 29 screened against acetylcholinesterase (AChE) and butyrylcholinesterase (BChE).

\begin{tabular}{ccc}
\hline \multirow{2}{*}{ Compound } & \multicolumn{2}{c}{ Inhibition $\left(\mathbf{\%} \pm\right.$ S.D. $\left.^{\mathbf{a}}\right)$ at $\mathbf{1 0 0 0} \boldsymbol{\mu \mathbf { M } ^ { \mathbf { b } }}$} \\
\cline { 2 - 3 } & AChE & BChE \\
\hline $\mathbf{1 3}$ & $19.33 \pm 3.03$ & $25.21 \pm 2.58$ \\
$\mathbf{2 6}$ & $80.53 \pm 1.22\left(\mathrm{IC}_{50}=294.18 \pm 5.26 \mu \mathrm{M}\right)$ & $48.46 \pm 2.29$ \\
$\mathbf{2 9}$ & $52.55 \pm 2.60\left(\mathrm{IC}_{50}=655.18 \pm 6.35 \mu \mathrm{M}\right)$ & $33.41 \pm 1.37$ \\
Reference $^{\mathrm{c}}$ & $97.92 \pm 0.01\left(\mathrm{IC}_{50}=2.29 \pm 0.33 \mu \mathrm{M}\right)$ & $91.52 \pm 1.63\left(\mathrm{IC}_{50}=124.03 \pm 4.05 \mu \mathrm{M}\right)$ \\
\hline & \multicolumn{2}{c}{${ }^{\text {a }}$ Standard deviation; ${ }^{\mathrm{b}}$ Final concentration; ${ }^{\mathrm{c}}$ Galantamine HBr. }
\end{tabular}

\subsection{Molecular Docking Simulations of Yuccalechins B and C}

To explore possible binding interactions of $\mathbf{2 6}$ and $\mathbf{2 9}$, molecular docking simulations were carried out on hAChE (PDB: 4EY7) using a Glide module implemented in the Schrödinger Small-Molecule Drug Discovery Suite. The results revealed that 26 and 29 exhibited binding energies of -8.43 and $-7.76 \mathrm{kcal} \mathrm{mol}^{-1}$, respectively, against AChE, which were modulated by hydrogen bonds and $\pi-\pi$ stacking contacts inside the active site.

According to molecular docking results, the orientation of $\mathbf{2 6}$ was driven by the interactions with the peripheral anionic site (PAS) comprising residues. Compound $\mathbf{2 6}$ was located in the bottleneck of the active site gorge via $\pi-\pi$ stacking contacts with Tyr341 and forming a hydrogen bond with Tyr72 at the entrance to the gorge through the 5-positioned hydroxyl group of the A-ring. The oxygen atom of the F-ring pointed toward the acyl binding site and was found to interact with the Phe295 backbone via hydrogen bonding. Moreover, the $\pi-\pi$ stacking contact between the E-ring and the oxyanion hole residue Phe338 stabilized the occupation of $\mathbf{2 6}$ in the binding site (Figure 9). The observation for $\mathbf{2 6}$ was found to be in good agreement with the data reported, indicating the aromatic residues at the PAS as a major site of interaction for polycyclic aromatic compounds [84].
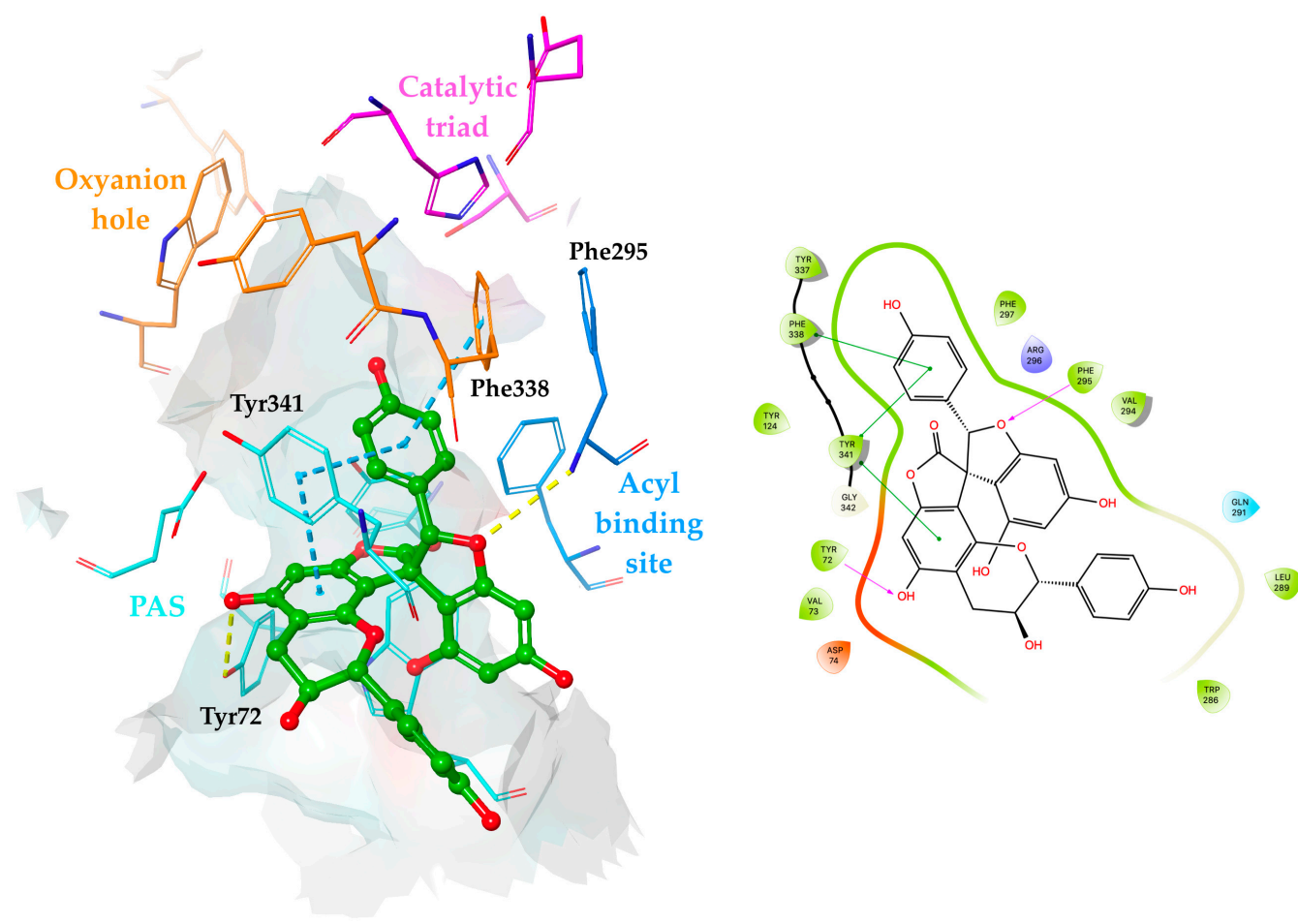

Figure 9. Proposed binding mode and 2D interaction map for $\mathbf{2 6}$ (presented as a green ball and stick model) in the hAChE active site (PDB: 4EY7). Hydrogen bonds and $\pi-\pi$ stacking contacts are represented by yellow and cyan dashed lines, respectively. 
As depicted in Figure 10, 29 was positioned deeply in the enzyme gorge occupying the region between the oxyanion hole and the acyl binding pocket, which was close to catalytic triad residues. The E-ring displayed interactions with His447 and Phe338 through hydrogen bonding and $\pi-\pi$ stacking contacts, respectively, which were found to be responsible for stabilization of 29 at the binding site.
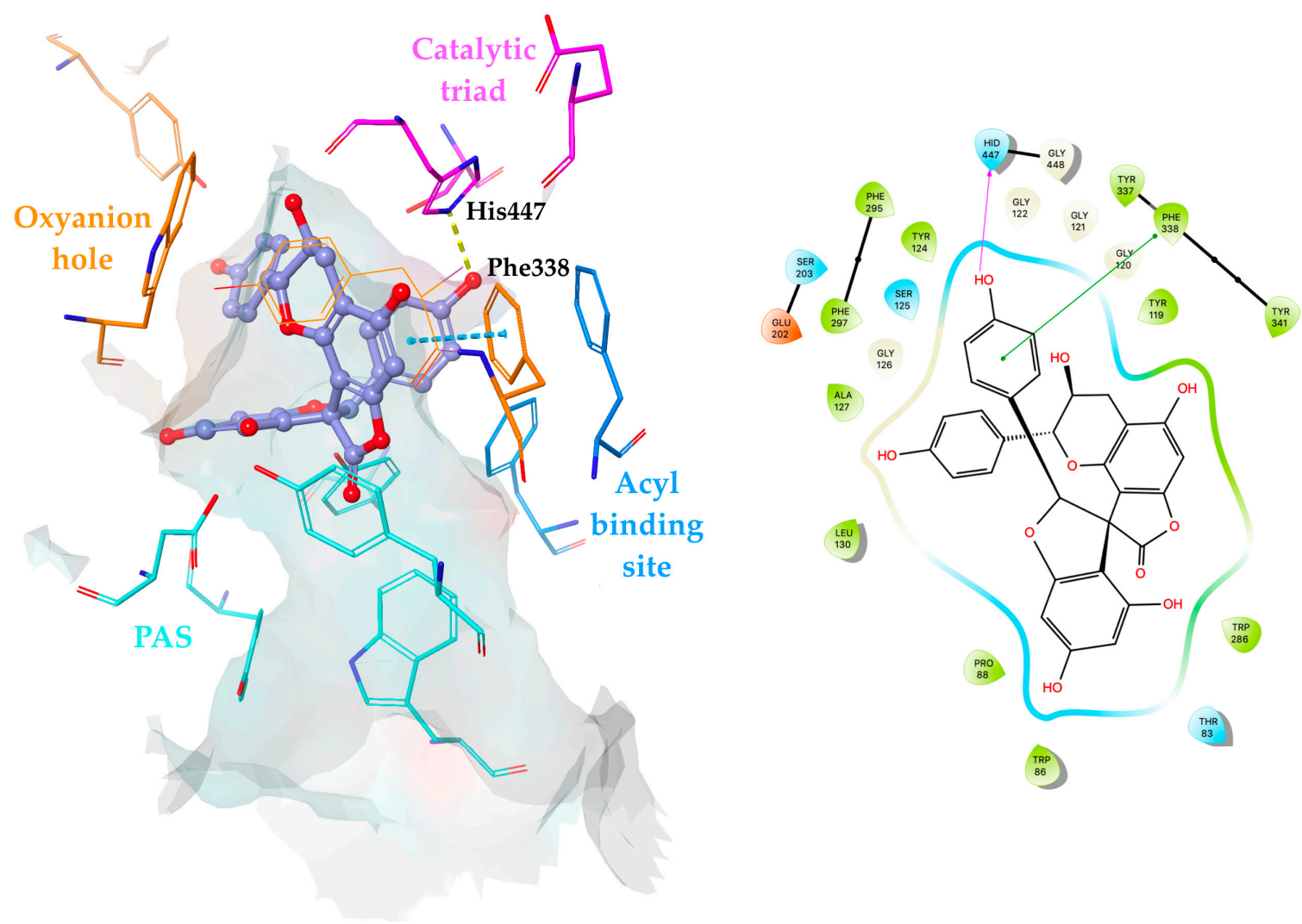

Figure 10. Proposed binding mode and 2D interaction map for 29 (presented as a purple ball and stick model) in the hAChE active site (PDB: 4EY7). Hydrogen bonds and $\pi-\pi$ stacking contacts are represented by yellow and cyan dashed lines, respectively.

Based on the results of the molecular docking simulations, the reduced number of favorable $\pi-\pi$ stacking and hydrogen bonding contacts with key residues, which tend to increase the binding affinity, might be the explanation for the lower inhibitory capacity of these compounds against AChE.

\section{Discussion}

The multistep purification of Yucca schidigera bark led to the isolation of three new spirobiflavonoids and confirmed the presence of numerous phenolic compounds, among which aromadendrin, naringenin, yuccalide A, and gloriosaols A and C-E are reported for the first time in this plant. Structures of isolated compounds were elucidated using various spectroscopic methods, including HRESIMS, UV, and ECD spectroscopy and optical rotations. For the new compounds, the relative configuration was established based on NMR chemical shifts, $\mathrm{H}-\mathrm{H}$ and $\mathrm{C}-\mathrm{H}$ coupling constants, DP4+ probability calculations, and NOE effects observed in the 2D-ROESY NMR spectra. Here, we report for the first time the usage of ${ }^{1} J_{\mathrm{CH}},{ }^{2} J_{\mathrm{CH}}$, and ${ }^{3} \mathrm{~J}_{\mathrm{CH}}$ coupling constants in the determination of relative stereochemistry of flavan-3-ols and spirobiflavonoids. Additionally, the absolute configuration of chiral spirobiflavonoids has been described for the first time using ab initio calculations of ECD spectra. The identification of stereochemistry of such compounds reported so far was based on a comparison of the ECD spectra with larixinol (abiesinol E), which possesses the $2 " R, 3$ " $R, 2 R, 3 R$ absolute configuration established by the X-ray crystallographic analysis [19], or chemical methods [20]. Our work, to the best of our 
knowledge, is the first report on the cholinesterase inhibitory activity of spirobiflavonoids. Tested compounds, yuccalechins B (26) and C (29), turned out very weak, but they were selective inhibitors of AChE.

\section{Materials and Methods}

\subsection{Chemicals and Reagents}

Methanol and chloroform as well as acetic acid, $n$-hexane, and ethyl acetate, all of analytical reagent grade, were purchased from Fisher Chemical (Loughborough, UK) and Merck (Darmstadt, Germany), respectively. Acetonitrile and methanol (LC-MS grade) were purchased from Merck (Darmstadt, Germany), while MS-grade formic acid and other chemicals were purchased from Sigma-Aldrich (Steinheim, Germany). Ultrapure water was prepared using a Milli-Q water purification system (Millipore, Milford, MA, USA).

\subsection{Plant Material}

Yucca schidigera Roezl ex Ortgies bark was purchased from the commercial source Desert King Int. (Chula Vista, CA, USA). A voucher specimen (No. IUNG-DBCQ-YS01) has been deposited in the Department of Biochemistry and Crop Quality, Institute of Soil Science and Plant Cultivation, State Research Institute, Poland.

\subsection{Extraction and Isolation}

The yucca bark was powdered using an Ultra Centrifugal Mill ZM 200 (Retsch, Germany) with $0.5 \mathrm{~mm}$ sieves, and then $202.8 \mathrm{~g}$ of the powder was extracted with $100 \% \mathrm{MeOH}(3 \mathrm{~L} \times 3,1$ day each) using an ultrasonic bath (Polsonic 33, Warsaw, Poland) at room temperature. All extraction, isolation, and separation procedures were performed in the dark to avoid any isomerization of compounds. Combined filtered solutions were concentrated under reduced pressure at $35^{\circ} \mathrm{C}$ followed by dilution with water and defatted with $n$-hexane in a separating funnel. The solution obtained was evaporated to eliminate $\mathrm{MeOH}$ and subsequently was extracted with ethyl acetate to yield $12.3 \mathrm{~g}$ of the EtOAc fraction after evaporation and lyophilization (Gamma 2-16 LSC freeze dryer, Martin Christ Gefriertrocknungsanlagen $\mathrm{GmbH}$, Germany). Part of the EtOAc fraction $(1.0 \mathrm{~g} \times 3)$ was separated by gel-filtration chromatography on a glass column $(100 \times 3.2 \mathrm{~cm}$ i.d., Millipore Corp., Bedford MA, USA) filled with Sephadex LH-20 (40-120 $\mu \mathrm{m}$, Sigma-Aldrich, Steinheim, Germany), eluted with MeOH at a flow rate of $2.5 \mathrm{~mL} \mathrm{~min}^{-1}$, and connected to a Gilson HPLC-ELSD apparatus (Gilson Inc., Middleton, WI, USA). As a result of this separation, 8 fractions (Fr. 1-8) were obtained. The fractions selected (Fr. 3-8) were subsequently purified by flash chromatography (FC) on silica gel cartridges $(25 \mu \mathrm{m}$, SNAP Ultra, Biotage, Uppsala, Sweden). The samples were dry-loaded onto cartridges and eluted in a stepwise gradient of acetone in the solvent mixtures of chloroform:acetone:acetic acid 75:1.65:8.5; 75:3.3:8.5; 75:16.5:8.5; and 75:24.75:8.5 [85] at a flow rate of 43-55 $\mathrm{mL} \mathrm{min}^{-1}$. Collected fractions (each $16 \mathrm{~mL}$ ) were combined according to thin layer chromatography (TLC) carried out on silica gel $60 \mathrm{~F}_{254 \mathrm{~S}}$ plates (Merck, Darmstadt, Germany) after visualization under UV light at 254/360 nm. The yielded subfractions were concentrated under reduced pressure and immediately submitted to solid phase extraction (SPE) performed on Oasis HLB 12 cc Vac cartridges (Waters Corp., Milford, MA, USA) for the elimination of acetic acid from the samples. Finally, individual compounds were purified using semi-preparative HPLC.

\subsection{Semi-Preparative HPLC}

Further purification of the FC fractions was carried out either on a Dionex chromatographic system (Dionex ${ }^{\mathrm{TM}}$, Sunnyvale, CA, USA) equipped with a PDA-100 detector, a P680 HPLC pump, an ASI-100 automated sample injector, a TCC-100 thermostatted column compartment, a Gilson FC 204 fraction collector (Gilson Inc., Middleton, WI, USA), or on a Gilson chromatographic system (Gilson 
Inc., Middleton, WI, USA) equipped with an evaporative light-scattering detector (ELSD, Gilson PrepELS II), a Gilson 321 HPLC pump, and a Gilson GX-271 liquid handler/fraction collector with a $2 \mathrm{~mL}$ sample loop. The columns used for separations included Kromasil 100-5-C18 $(25 \times 1.0 \mathrm{~cm}$ i.d., $5 \mu \mathrm{m}$, AkzoNobel, Bohus, Sweden), column \#1; Cosmosil $\pi$ NAP $(25 \times 1.0 \mathrm{~cm}$ i.d., $5 \mu \mathrm{m}$, Nacalai Tesque, INC., Kyoto, Japan), column \#2; and Atlantis Prep T3 $(25 \times 1.0 \mathrm{~cm}$ i.d., $5 \mu \mathrm{m}$, Waters, Milford, MA), column \#3. The separation protocol was individually improved for each fraction. Semi-preparative HPLC analyses were carried out using isocratic or gradient conditions with aqueous acetonitrile or methanol solutions containing $0.1 \%$ formic acid (FA). The mobile phase flow rate ranged from 3.4 to $4.0 \mathrm{~mL} \mathrm{~min}^{-1}$, and the columns were held at $35-50^{\circ} \mathrm{C}$. The separation of subfractions SFr. $4-5$ in column $\# 1$, at $40{ }^{\circ} \mathrm{C}$ in an isocratic mode of $\mathrm{MeCN} / \mathrm{H}_{2} \mathrm{O} / \mathrm{FA}(21: 79: 0.1, v / v / v)$ at a flow rate of $4.0 \mathrm{~mL} \mathrm{~min}^{-1}$, afforded compounds 15 (yuccalechin A, $1.67 \mathrm{mg}$ ), 26 (yuccalechin B, $14.3 \mathrm{mg}$ ), and 29 (yuccalechin C, $13.34 \mathrm{mg}$ ); SFr. 3-2 yielded compound 16 (aromadendrin, $9.4 \mathrm{mg}$ ); SFr. 4-1 yielded compound 38 (naringenin, $23.02 \mathrm{mg}$ ); SFr. 5-3 yielded compound 13 (trans-3,3',5,5'-tetrahydroxy-4' -methoxystilbene, $23.21 \mathrm{mg}$ ); and Fr. 5-1 yielded compound 21 (trans-resveratrol, $13.86 \mathrm{mg}$ ). The most abundant, Fr. 6 (1012 mg), was divided into 7 subfractions, and SFr. 6-1 afforded compound 44 (kaempferol, $7.21 \mathrm{mg}$ ), SFr. 6-4 afforded 47 (yuccaol A, $9.96 \mathrm{mg}$ ) and 48 (yuccaol B, $10.1 \mathrm{mg}$ ), SFr. 6-5 afforded 37 (yuccaol E, $38.7 \mathrm{mg}$ ), 39 (yuccaol C, $116.22 \mathrm{mg}$ ), and 42 (yuccaol D, $63.92 \mathrm{mg}$ ), SFr. 6-6 afforded 40 (yuccalide A, $3.64 \mathrm{mg}$ ), and SFr. 6-7 afforded 49 (gloriosaol E, $19.94 \mathrm{mg}$ ) and 50 (gloriosaol D, $19.31 \mathrm{mg}$ ). Lastly, Fr. 7 and 8 yielded molecules 54 (gloriosaol A, 14.63) and 58 (gloriosaol C, $11.08 \mathrm{mg}$ ), respectively. ${ }^{1} \mathrm{H}$ - and ${ }^{13} \mathrm{C}$-NMR spectra of all isolated compounds are available in the Supplementary Materials. Prior to the biological activity assays, the purities of compounds 13, 26, and 29 were checked by NMR spectroscopy (electronic reference to access in vivo concentrations 2 (ERETIC2) method) [86] using D-(-)-quinic acid $\left(\mathrm{C}_{7} \mathrm{H}_{12} \mathrm{O}_{6}, 98 \%\right.$, Sigma-Aldrich, St. Louis, MO, USA) in deuterated methanol $(30 \mathrm{mM})$ as the reference sample. The purity was over $60 \%$ for all compounds tested.

\subsection{High-Resolution LC-MS}

The EtOAc fraction was subjected to high-resolution LC-MS analysis. Chromatographic separation was performed on a Thermo Scientific Ultimate 3000RS chromatographic system on a Waters BEH C18 column $(150 \times 2.1 \mathrm{~mm}$ i.d.; $1.7 \mu \mathrm{m}$, Milford, USA). The effluent was analyzed using a photodiode array detector (200-600 nm, $10 \mathrm{~Hz}$ acquisition frequency), Q-TOF MS (Bruker Impact II HD, Bruker, Billerica, MA, USA), and a charged aerosol detector (CAD, Thermo Corona Veo RS) as described in detail in our previous publication [87].

\subsection{NMR Spectroscopy}

The 1D and 2D NMR spectra $\left({ }^{1} \mathrm{H},{ }^{13} \mathrm{C}\right.$ DEPTQ,${ }^{1} \mathrm{H}-{ }^{13} \mathrm{C}$ HSQC,,${ }^{1} \mathrm{H}-{ }^{13} \mathrm{C}$ H2BC, ${ }^{1} \mathrm{H}^{-13} \mathrm{C}$ HMBC, ${ }^{1} \mathrm{H}-{ }^{13} \mathrm{C}$ F2-coupled HSQC, ${ }^{1} \mathrm{H}-{ }^{13} \mathrm{C}$ HSQC-HECADE, ${ }^{1} \mathrm{H}-{ }^{1} \mathrm{H}$ COSY, and ${ }^{1} \mathrm{H}-{ }^{1} \mathrm{H}$ ROESY) were recorded using an Avance III HD Ascend $500 \mathrm{MHz}$ spectrometer (Bruker BioSpin, Rheinstetten, Germany) in deuterated methanol $\left(\mathrm{MeOH}-d_{4}\right)$ at $30^{\circ} \mathrm{C}$. NMR spectra were calibrated to the signal of residual solvent: $\delta 3.31$ for ${ }^{1} \mathrm{H}$ and 49.0 for ${ }^{13} \mathrm{C}$.

\subsection{Optical Rotation $[\alpha]$}

Optical rotations were determined on a P-2000 polarimeter (Jasco, Easton, PA, USA) in $\mathrm{MeOH}$ solutions at concentrations of $1 \mathrm{mg} \mathrm{mL}^{-1}$.

\subsection{Electronic Circular Dichroism (ECD) Spectroscopy}

Circular dichroism spectra of all analyzed compounds were made at room temperature on a Jasco $\mathrm{J}-1500$ magnetic circular dichroism spectrometer ( $1 \mathrm{~mm}$ or $5 \mathrm{~mm}$ pathlength was used) in $\mathrm{MeOH}$. CD spectra were collected at a scan rate of $100 \mathrm{~nm} \mathrm{~min}^{-1}$ with a response time of $1 \mathrm{~s}$. Measurements were taken in the $200-500 \mathrm{~nm}$ range. All spectra were baseline corrected, and the final plot was taken from five accumulated plots. The concentrations of compounds were $50 \mathrm{mM}$. 


\subsection{Enzyme Inhibition Assay}

$\mathrm{AChE}$ and BChE inhibitory activities were measured by a slightly modified spectrophotometric method of Ellman et al. [81]. Electric eel AChE (Type-VI-S, EC 3.1.1.7, Sigma, St. Louis, MO, USA) and horse serum BChE (EC 3.1.1.8, Sigma, St. Louis, MO, USA) were used as the enzyme sources, while acetylthiocholine iodide and butyrylthiocholine chloride (Sigma, St. Louis, MO, USA) were employed as the substrates of the reaction. 5,5'-Dithio-bis(2-nitrobenzoic) acid (DTNB, Sigma, St. Louis, MO, USA) was used to measure the anticholinesterase activity as the coloring agent. All reagents and conditions were exactly the same as described in our previous publication [88]. Hydrolysis of acetylthiocholine iodide/butyrylthiocholine chloride was monitored by the formation of the yellow 5-thio-2-nitrobenzoate anion, as a result of the reaction of DTNB with thiocholines, catalyzed by enzymes at $412 \mathrm{~nm}$ utilizing a 96-well microplate reader (VersaMax Molecular Devices, USA). The measurements and calculations were evaluated by using Softmax PRO 4.3.2.LS software. The percent inhibitions of $\mathrm{AChE} / \mathrm{BChE}$ were determined by comparing the reaction rates of the samples relative to a blank sample (ethanol in phosphate buffer $\mathrm{pH}=8$ ), using the formula $(\mathrm{E}-\mathrm{S}) / \mathrm{E} \times 100$, where $\mathrm{E}$ is the activity of enzyme without test sample, and $\mathrm{S}$ is the activity of the enzyme with the test sample. The experiments were conducted in six replicates. Galantamine (Sigma, St. Louis, MO, USA) was used as the reference.

\subsection{DP4+ Probability Calculation}

Intended compounds were subjected to conformational analysis by MacroModel 9.1 (Schrödinger. LLC, New York, NY, USA) using an OPLS-3 force field in $\mathrm{H}_{2} \mathrm{O}$. Geometrical optimization of using DFT/6-31G(d) in the gas phase (compound 29) or DFT/6-31G(d,p)/IEFPCM/methanol (compound 15 and 26) in Gaussian 16 was performed [89]. Subsequently, they were submitted to NMR chemical shift calculations using the gauge-independent atomic orbitals (GIAOs) method in rmpw1pw91/6-311G+(d,p)/IEFPCM/methanol. The shift tensors obtained were further adjusted to chemical shifts by using TMS proton and carbon chemical shifts, which were calculated using the same method. All chemical shifts were Boltzmann-averaged, and unscaled chemical shifts were used for the DP4+ probability calculation based on the method and the interactive Excel sheet published by Grimblat et al. [51]

\subsection{ECD Spectra Calculation}

3D structures of isolated compounds were drawn in Maestro (Schrödinger. LLC, New York, NY, USA) and subjected to conformational analysis using MacroModel 9.1 (Schrödinger. LLC, New York, NY, USA) and OPLS-3 as a force field in $\mathrm{H}_{2} \mathrm{O}$. Geometrical optimization and energy calculations of conformers occurring in the energy window of $5 \mathrm{kcal} \mathrm{mol}^{-1}$ were performed by implementing DFT/ 6-31G(d) in the gas phase for compound 29 or DFT/6-31G(d,p)/ IEFPCM/methanol for compound 26 and 29. Subsequently, ECD spectra of optimized compounds were simulated by using TD-DFT/ B3LYP/6-31G(d,p)/IEFPCM/methanol (compound 15) or TD-DFT/cam-B3LYP/6-31G(d,p)/IEFPCM/ methanol (compound 26 and 29). ECD spectra obtained (with a half-band of $0.2-0.3 \mathrm{eV}$ ) were Boltzmann-averaged, and a UV correction of +10 to $+25 \mathrm{~nm}$ was applied to compare them with experimental spectra obtained in methanol.

\subsection{Molecular Docking Studies}

Molecular docking studies were carried out using the induced fit docking (IFD) protocol implemented in the Schrödinger Small-Molecule Drug Discovery Suite (Small-Molecule Drug Discovery Suite 2019-3, Schrödinger, LLC, New York, NY, 2019). The compounds built via a builder panel in Maestro were subjected to ligand preparation by LigPrep (Schrödinger Release 2019-3: LigPrep, Schrödinger, LLC, New York, NY, 2019) using default conditions. The crystal structure of hAChE (PDB: 4EY7) [90] was retrieved from the Protein Data Bank. The protein was prepared using the 
protein preparation wizard tool. Water molecules were removed from the crystallographic structure followed by the addition of hydrogen atoms. All atom charges and atom types were assigned. Finally, energy minimization and refinement of the structure was performed up to $0.3 \AA$ RMSD by applying an OPLS3e force field. The centroid of the active site residues was defined as a grid box. Van der Waals $(\mathrm{vdW})$ radius scaling factor 1.00, partial charge cutoff 0.25 , and an OPLS3e force field were used for receptor grid generation. The compounds prepared by LigPrep were docked into AChE using the IFD protocol [91], which considers flexibility of both the compounds and receptor. Residues Asp74, Trp86, Tyr124, Tyr133, Ser203, Trp286, Phe295, Phe297, Try337, Phe338, and His447 lining the binding site of AChE were kept as flexible. The initial docking protocol was set to employ a $0.50 \mathrm{vdW}$ radius scaling factor, and the resulting top 20 poses of each compound were taken. An extra-precision (XP) algorithm was employed in redocking of the compounds, with the low energy refined structures generated by the Prime MM-GBSA (molecular mechanics - generalized Born surface area) method. The best conformation for each compound was chosen based on the lowest XP glide score.

Supplementary Materials: Supplementary Materials are available online.

Author Contributions: Conceptualization, investigation, methodology, compound purification, NMR data acquisition, NMR and MS data interpretation, writing-original draft preparation, Ł.P.; formal analysis, NMR data interpretation, DP4+ and ECD calculations, writing-original draft preparation, visualization, M.A.; investigation, compound purification, writing — original draft preparation, S.K.; investigation, AChE/BChE activity measurements and interpretation, I.E.O. and F.S.S.D.; formal analysis, molecular docking studies, visualization, G.E.; supervision, resources, funding acquisition, H.S. and W.O.

Funding: This research was funded by European Commission program HORIZON 2020, call: H2020 WIDESPREAD-2014-2, topic: ERA Chairs, grant number 669062.

Acknowledgments: The authors would like to acknowledge Mariusz Kowalczyk, Department of Biochemistry and Crop Quality, Institute of Soil Science and Plant Cultivation (Poland), for carrying out LC-MS data acquisition; Aleksandra Marciniak, Department of Inorganic Chemistry, Wroclaw Medical University (Poland), for measuring ECD spectra; and Sylwester Ślusarczyk, Department of Pharmaceutical Biology and Botany, Wroclaw Medical University (Poland), for carrying out ECD and optical rotation measurements. The computational results presented have been achieved (in part) using the HPC infrastructure LEO of the University of Innsbruck.

Conflicts of Interest: The authors declare no conflicts of interest.

\section{References}

1. Ortgies, K.E. Gartenflora. Monatschrift für deutsche und schweizerische Garten-und Blumenkunde; Verlag von Ferdinand Enke: Erlangen, Germany, 1871. Available online: https://www.biodiversitylibrary.org/item/125737 (accessed on 7 October 2019).

2. Cheeke, P.R. Saponins: Surprising Benefit of Desert Plants; Linus Pauling Institute Newsletter Oregon State University: Corvallis, OR, USA, 1998; pp. 4-5.

3. Cheeke, P.R. Actual and Potential Applications of Yucca Schidigera and Quillaja Saponaria Saponins in Human and Animal Nutrition. In Saponins in Food, Feedstuffs and Medicinal Plants; Springer: Dordrecht, The Netherlands, 2000; pp. 241-254.

4. Miyakoshi, M.; Tamura, Y.; Masuda, H.; Mizutani, K.; Tanaka, O.; Ikeda, T.; Ohtani, K.; Kasai, R.; Yamasaki, K. Antiyeast Steroidal Saponins from Yucca schidigera (Mohave Yucca), a New Anti-Food-Deteriorating Agent. J. Nat. Prod. 2000, 63, 332-338. [CrossRef]

5. Oleszek, W.; Sitek, M.; Stochmal, A.; Piacente, S.; Pizza, C.; Cheeke, P. Steroidal saponins of Yucca schidigera Roezl. J. Agric. Food Chem. 2001, 49, 4392-4396. [CrossRef] [PubMed]

6. Kowalczyk, M.; Pecio, Ł.; Stochmal, A.; Oleszek, W. Qualitative and Quantitative Analysis of Steroidal Saponins in Crude Extract and Bark Powder of Yucca schidigera Roezl. J. Agric. Food Chem. 2011, 59, 8058-8064. [CrossRef] [PubMed]

7. Piacente, S.; Montoro, P.; Oleszek, W.; Pizza, C. Yucca schidigera Bark: Phenolic Constituents and Antioxidant Activity. J. Nat. Prod. 2004, 67, 882-885. [CrossRef] [PubMed]

8. Montoro, P.; Piacente, S.; Oleszek, W.; Pizza, C. Liquid chromatography/tandem mass spectrometry of unusual phenols from Yucca schidigera bark: Comparison with other analytical techniques. J. Mass Spectrom. 2004, 39, 1131-1138. [CrossRef] [PubMed] 
9. Oleszek, W.; Sitek, M.; Stochmal, A.; Piacente, S.; Pizza, C.; Cheeke, P. Resveratrol and Other Phenolics from the Bark of Yucca schidigera Roezl. J. Agric. Food Chem. 2001, 49, 747-752. [CrossRef] [PubMed]

10. Piacente, S.; Bifulco, G.; Pizza, C.; Stochmal, A.; Oleszek, W. A novel phenolic spiro derivative, Yuccaone A, from Yucca schidigera bark. Tetrahedron Lett. 2002, 43, 9133-9136. [CrossRef]

11. Fedorova, T.E.; Ivanova, S.Z.; Babkin, V.A. Spiroflavonoid compounds: Structure and distribution in nature review. Russ. J. Bioorgan. Chem. 2010, 36, 793-801. [CrossRef]

12. Wenzig, E.M.; Oleszek, W.; Stochmal, A.; Kunert, O.; Bauer, R. Influence of Phenolic Constituents from Yucca schidigera Bark on Arachidonate Metabolism in Vitro. J. Agric. Food Chem. 2008, 56, 8885-8890. [CrossRef]

13. Bassarello, C.; Bifulco, G.; Montoro, P.; Skhirtladze, A.; Benidze, M.; Kemertelidze, E.; Pizza, C.; Piacente, S. Yucca gloriosa: A Source of Phenolic Derivatives with Strong Antioxidant Activity. J. Agric. Food Chem. 2007, 55, 6636-6642. [CrossRef]

14. Teponno, R.B.; Ponou, B.K.; Fiorini, D.; Barboni, L.; Tapondjou, L.A. Chemical Constituents from the Roots of Furcraea bedinghausii Koch. Int. Lett. Chem. Phys. Astron. 2013, 11, 9-19. [CrossRef]

15. Nakashima, K.I.; Abe, N.; Oyama, M.; Inoue, M. Yuccalides A-C, three new phenolic compounds with spiro-structures from the roots of Yucca gloriosa. Fitoterapia 2016, 111, 154-159. [CrossRef] [PubMed]

16. Shen, Z.; Haslam, E.; Falshaw, C.P.; Begley, M.J. Procyanidins and polyphenols of Larix gmelini bark. Phytochemistry 1986, 25, 2629-2635. [CrossRef]

17. Taniguchi, M.; Fujiwara, A.; Baba, K.; Wang, N.-H. Two biflavonoids from Daphne acutiloba. Phytochemistry 1998, 49, 863-867. [CrossRef]

18. Yang, B.-H.; Zhang, W.-D.; Liu, R.-H.; Tan, C.-H.; Li, T.-Z.; Zhang, C.; Xu, X.-K.; Su, J. Spiro-biflavonoids from Larix olgensis Henry var. koreana Nakai. Helv. Chim. Acta 2005, 88, 2892-2896. [CrossRef]

19. Li, Y.L.; Yang, X.W.; Li, S.M.; Tang, J.; Tian, J.M.; Peng, X.Y.; Huang, D.S.; Zhang, W.D. Two new spirobiflavonoids from Abies chensiensis with moderate NO production inhibitory activity. Planta Med. 2009, 75, 1534-1537. [CrossRef]

20. Wada, S.; Hitomi, T.; Tanaka, R. Phenolic Compounds Isolated from the Bark of Abies sachalinensis. Helv. Chim. Acta 2009, 92, 1610-1620. [CrossRef]

21. Buckingham, J.; Munasinghe, V.R.N. Dictionary of Flavonoids with CD-ROM; CRC Press: Boca Raton, FL, USA, 2015; ISBN 9781482282504.

22. Dementia. Available online: https://www.who.int/news-room/fact-sheets/detail/dementia (accessed on 7 October 2019).

23. Dolan, D.; Troncoso, J.; Resnick, S.M.; Crain, B.J.; Zonderman, A.B.; OșBrien, R.J. Age, Alzheimer's disease and dementia in the Baltimore Longitudinal Study of Ageing. Brain 2010, 133, 2225-2231. [CrossRef]

24. Orhan, G.; Orhan, I.; Subutay-Oztekin, N.; Ak, F.; Sener, B. Contemporary anticholinesterase pharmaceuticals of natural origin and their synthetic analogues for the treatment of Alzheimer's disease. Recent Pat. CNS Drug Discov. 2009, 4, 43-51. [CrossRef]

25. Tundis, R.; Loizzo, M.R.; Nabavi, S.M.; Orhan, I.E.; Skalicka-Woźniak, K.; D’Onofrio, G.; Aiello, F. Natural Compounds and Their Derivatives as Multifunctional Agents for the Treatment of Alzheimer Disease. In Discovery and Development of Neuroprotective Agents from Natural Products; Elsevier: Amsterdam, The Netherlands, 2018; pp. 63-102, ISBN 9780128097694.

26. Abbas-Mohammadi, M.; Moridi, M.; Salehi, P.; Nejad, S.E.; Sonboli, A.; Kelso, C.; Skropeta, D. Acetylcholinesterase-inhibitory activity of Iranian plants: Combined HPLC/bioassay-guided fractionation, molecular networking and docking strategies for the dereplication of active compounds. J. Pharm. Biomed. Anal. 2018, 158, 471-479. [CrossRef]

27. Brus, B.; Košak, U.; Turk, S.; Pišlar, A.; Coquelle, N.; Kos, J.; Stojan, J.; Colletier, J.-P.; Gobec, S. Discovery, Biological Evaluation, and Crystal Structure of a Novel Nanomolar Selective Butyrylcholinesterase Inhibitor. J. Med. Chem. 2014, 57, 8167-8179. [CrossRef] [PubMed]

28. Musial, A.; Bajda, M.; Malawska, B. Recent Developments in Cholinesterases Inhibitors for Alzheimers Disease Treatment. Curr. Med. Chem. 2007, 14, 2654-2679. [CrossRef] [PubMed]

29. Ferris, S.H. Switching previous therapies for Alzheimer's disease to galantamine. Clin. Ther. 2001, 23, A3-A7. [CrossRef]

30. Qian, Z.M.; Ke, Y. Huperzine A: Is it an Effective Disease-Modifying Drug for Alzheimer's Disease? Front. Aging Neurosci. 2014, 6, 216. [CrossRef] 
31. Khaw, K.Y.; Choi, S.B.; Tan, S.C.; Wahab, H.A.; Chan, K.L.; Murugaiyah, V. Prenylated xanthones from mangosteen as promising cholinesterase inhibitors and their molecular docking studies. Phytomedicine 2014, 21, 1303-1309. [CrossRef]

32. Orhan, I.E. Implications of Some Selected Flavonoids Towards Alzheimer's Disease with the Emphasis on Cholinesterase Inhibition and their Bioproduction by Metabolic Engineering. Curr. Pharm. Biotechnol. 2014, 15, 352-361. [CrossRef]

33. Chen, X.; Mukwaya, E.; Wong, M.-S.; Zhang, Y. A systematic review on biological activities of prenylated flavonoids. Pharm. Biol. 2014, 52, 655-660. [CrossRef]

34. Al-Gubory, K.H.; Laher, I. (Eds.) Nutritional Antioxidant Therapies: Treatments and Perspectives; Springer International Publishing: Cham, Switzerland, 2017; ISBN 978-3-319-67623-4.

35. Viña, J.; Lloret, A.; Ortí, R.; Alonso, D. Molecular bases of the treatment of Alzheimer's disease with antioxidants: Prevention of oxidative stress. Mol. Asp. Med. 2004, 25, 117-123. [CrossRef]

36. Zhu, X.; Raina, A.K.; Lee, H.; Casadesus, G.; Smith, M.A.; Perry, G. Oxidative stress signalling in Alzheimer's disease. Brain Res. 2004, 1000, 32-39. [CrossRef]

37. Orhan, I.; Kartal, M.; Naz, Q.; Ejaz, A.; Yilmaz, G.; Kan, Y.; Konuklugil, B.; Şener, B.; Iqbal Choudhary, M. Antioxidant and anticholinesterase evaluation of selected Turkish Salvia species. Food Chem. 2007, 103, 1247-1254. [CrossRef]

38. Jang, M.H.; Piao, X.L.; Kim, J.M.; Kwon, S.W.; Park, J.H. Inhibition of cholinesterase and amyloid- $\beta$ aggregation by resveratrol oligomers from Vitis amurensis. Phyther. Res. 2008, 22, 544-549. [CrossRef] [PubMed]

39. Pan, L.-F.; Wang, X.-B.; Xie, S.-S.; Li, S.-Y.; Kong, L.-Y. Multitarget-directed resveratrol derivatives: Anti-cholinesterases, anti- $\beta$-amyloid aggregation and monoamine oxidase inhibition properties against Alzheimer's disease. Medchemcomm 2014, 5, 609. [CrossRef]

40. Gal, J. Pasteur and the art of chirality. Nat. Chem. 2017, 9, 604-605. [CrossRef] [PubMed]

41. Mason, S. The origin of chirality in nature. Trends Pharmacol. Sci. 1986, 7, 20-23. [CrossRef]

42. Maier, M.E. Structural revisions of natural products by total synthesis. Nat. Prod. Rep. 2009, 26, 1105-1124. [CrossRef] [PubMed]

43. Nicolaou, K.C.; Shah, A.A.; Korman, H.; Khan, T.; Shi, L.; Worawalai, W.; Theodorakis, E.A. Total Synthesis and Structural Revision of Antibiotic CJ-16,264. Angew. Chemie Int. Ed. 2015, 54, 9203-9208. [CrossRef] [PubMed]

44. Li, Y. Structural revision of glabramycins B and C, antibiotics from the fungus Neosartorya glabra by DFT calculations of NMR chemical shifts and coupling constants. RSC Adv. 2015, 5, 36858-36864. [CrossRef]

45. Burns, D.C.; Mazzola, E.P.; Reynolds, W.F. The role of computer-assisted structure elucidation (CASE) programs in the structure elucidation of complex natural products. Nat. Prod. Rep. 2019, 36, 919-933. [CrossRef]

46. Smith, S.G.; Goodman, J.M. Assigning Stereochemistry to Single Diastereoisomers by GIAO NMR Calculation: The DP4 Probability. J. Am. Chem. Soc. 2010, 132, 12946-12959. [CrossRef]

47. Bagno, A.; Rastrelli, F.; Saielli, G. Toward the Complete Prediction of the ${ }^{1} \mathrm{H}$ and ${ }^{13} 3 \mathrm{C}$ NMR Spectra of Complex Organic Molecules by DFT Methods: Application to Natural Substances. Chem. A Eur. J. 2006, 12, 5514-5525. [CrossRef]

48. Bagno, A. Complete Prediction of the ${ }^{1} \mathrm{H}$ NMR Spectrum of Organic Molecules by DFT Calculations of Chemical Shifts and Spin-Spin Coupling Constants. Chemistry 2001, 7, 1652-1661.

49. Barone, G.; Duca, D.; Silvestri, A.; Gomez-Paloma, L.; Riccio, R.; Bifulco, G. Determination of the Relative Stereochemistry of Flexible Organic Compounds by Ab Initio Methods: Conformational Analysis and Boltzmann-Averaged GIAO ${ }^{13}$ C NMR Chemical Shifts. Chem. A Eur. J. 2002, 8, 3240. [CrossRef]

50. Barone, G.; Gomez-Paloma, L.; Duca, D.; Silvestri, A.; Riccio, R.; Bifulco, G. Structure Validation of Natural Products by Quantum-Mechanical GIAO Calculations of ${ }^{13} \mathrm{C}$ NMR Chemical Shifts. Chem. A Eur. J. 2002, 8, 3233. [CrossRef]

51. Grimblat, N.; Zanardi, M.M.; Sarotti, A.M. Beyond DP4: An Improved Probability for the Stereochemical Assignment of Isomeric Compounds using Quantum Chemical Calculations of NMR Shifts. J. Org. Chem. 2015, 80, 12526-12534. [CrossRef] [PubMed]

52. Nugroho, A.E.; Morita, H. Circular dichroism calculation for natural products. J. Nat. Med. 2014, 68, 1-10. [CrossRef] [PubMed] 
53. Bringmann, G.; Bruhn, T.; Maksimenka, K.; Hemberger, Y. The Assignment of Absolute Stereostructures through Quantum Chemical Circular Dichroism Calculations. Eur. J. Org. Chem. 2009, 2009, 2717-2727. [CrossRef]

54. Crawford, T.D.; Tam, M.C.; Abrams, M.L. The Current State of Ab Initio Calculations of Optical Rotation and Electronic Circular Dichroism Spectra. J. Phys. Chem. A 2007, 111, 12057-12068. [CrossRef]

55. Bassarello, C.; Bifulco, G.; Montoro, P.; Skhirtladze, A.; Kemertelidze, E.; Pizza, C.; Piacente, S. Gloriosaols A and B, two novel phenolics from Yucca gloriosa: Structural characterization and configurational assignment by a combined NMR-quantum mechanical strategy. Tetrahedron 2007, 63, 148-154. [CrossRef]

56. Sun, J.; Liang, F.; Bin, Y.; Li, P.; Duan, C. Screening Non-colored Phenolics in Red Wines using Liquid Chromatography/Ultraviolet and Mass Spectrometry/Mass Spectrometry Libraries. Molecules 2007, 12, 679-693. [CrossRef]

57. Xiao, H.; Parkin, K.L. Isolation and identification of potential cancer chemopreventive agents from methanolic extracts of green onion (Allium cepa). Phytochemistry 2007, 68, 1059-1067. [CrossRef]

58. Simmler, C.; Antheaume, C.; André, P.; Bonté, F.; Lobstein, A. Glucosyloxybenzyl Eucomate Derivatives from Vanda teres Stimulate HaCaT Cytochrome $c$ Oxidase. J. Nat. Prod. 2011, 74, 949-955. [CrossRef] [PubMed]

59. Cerezo, A.B.; Espartero, J.L.; Winterhalter, P.; Garcia-Parrilla, M.C.; Troncoso, A.M. (+)-Dihydrorobinetin: A Marker of Vinegar Aging in Acacia (Robinia pseudoacacia) Wood. J. Agric. Food Chem. 2009, 57, 9551-9554. [CrossRef] [PubMed]

60. Mabry, T.J.; Markham, K.R.; Thomas, M.B. The Systematic Identification of Flavonoids; Springer: Berlin/Heidelberg, Germany, 1970; ISBN 978-3-642-88460-3.

61. Rogerio-Candelera, M.A. Science, Technology and Cultural Heritage; CRC Press: London, UK, 2014; ISBN 9781315712420.

62. Zhang, J.; Xu, W.; Wang, P.; Huang, J.; Bai, J.; Huang, Z.; Liu, X.; Qiu, X. Chemical Analysis and Multi-Component Determination in Chinese Medicine Preparation Bupi Yishen Formula Using Ultra-High Performance Liquid Chromatography With Linear Ion Trap-Orbitrap Mass Spectrometry and Triple-Quadrupole Tandem Mass Spectrometry. Front. Pharmacol. 2018, 9, 568. [CrossRef]

63. Mari, A.; Montoro, P.; Pizza, C.; Piacente, S. Liquid chromatography tandem mass spectrometry determination of chemical markers and principal component analysis of Vitex agnus-castus L. fruits (Verbenaceae) and derived food supplements. J. Pharm. Biomed. Anal. 2012, 70, 224-230. [CrossRef] [PubMed]

64. Cuyckens, F.; Claeys, M. Mass spectrometry in the structural analysis of flavonoids. J. Mass Spectrom. 2004, 39, 1-15. [CrossRef] [PubMed]

65. Montoro, P.; Skhirtladze, A.; Bassarello, C.; Perrone, A.; Kemertelidze, E.; Pizza, C.; Piacente, S. Determination of phenolic compounds in Yucca gloriosa bark and root by LC-MS/MS. J. Pharm. Biomed. Anal. 2008, 47, 854-859. [CrossRef] [PubMed]

66. Min, K.; Hwang, B.; Lim, H.-S.; Kang, B.-S.; Oh, G.-J.; Lee, J.; Kang, S.-H.; Lee, K.; Ro, J.; Kim, Y. (-)-Epiafzelechin: Cyclooxygenase-1 Inhibitor and Anti-Inflammatory Agent from Aerial Parts of Celastrus orbiculatus. Planta Med. 1999, 65, 460-462. [CrossRef]

67. Shen, Z.; Falshaw, C.P.; Haslam, E.; Begley, M.J. A Novel Spiro-Biflavonoid from Larix gmelini. J. Chem. Soc. Chem. Commun. 1985, 1135. [CrossRef]

68. Hemingway, R.W.; Tobiason, F.L.; Wayne McGraw, G.; Steynberg, J.P. Conformation and Complexation of Tannins: NMR Spectra and Molecular Search Modeling of Flavan-3-ols. Magn. Reson. Chem. 1996, 34, 424-433. [CrossRef]

69. Kríž, Z.; Koča, J.; Imberty, A.; Charlot, A.; Auzély-Velty, R. Investigation of the complexation of (+)-catechin by $\beta$-cyclodextrin by a combination of NMR, microcalorimetry and molecular modeling techniques. Org. Biomol. Chem. 2003, 1, 2590-2595. [CrossRef]

70. Clark-Lewis, J.; Jackman, L.; Spotswood, T. Nuclear magnetic resonance spectra, stereochemistry, and conformation of flavan derivatives. Aust. J. Chem. 1964, 17, 632. [CrossRef]

71. Usman, A.; Thoss, V.; Nur-e-Alam, M. Isolation of (-)-Epicatechin from Trichilia emetica Whole Seeds. Am. J. Org. Chem. 2016, 21, 77-82.

72. Perlin, A.S.; Casu, B. Carbon-13 and proton magnetic resonance spectra of D-glucose- ${ }^{13}$ C. Tetrahedron Lett. 1969, 10, 2921-2924. [CrossRef]

73. Juaristi, E.; Cuevas, G. Manifestations of Stereoelectronic Interactions in ${ }^{1} \mathrm{~J}_{\mathrm{C}-\mathrm{H}}$ One-Bond Coupling Constants. Acc. Chem. Res. 2007, 40, 961-970. [CrossRef] 
74. Alabugin, I.V.; Manoharan, M.; Zeidan, T.A. Homoanomeric Effects in Six-Membered Heterocycles. J. Am. Chem. Soc. 2003, 125, 14014-14031. [CrossRef]

75. Alabugin, I.V. Stereoelectronic Interactions in Cyclohexane, 1, 3-Dioxane, 1, 3-Oxathiane, and 1, 3-Dithiane: W-Effect, $\sigma_{\mathrm{C}-\mathrm{X}} \leftrightarrow \sigma^{*} \mathrm{CH}$ Interactions, Anomeric Effect What Is Really Important? J. Org. Chem. 2000, 65, 3910-3919. [CrossRef]

76. Crews, P.; Rodriguez, J.; Jaspars, M. Organic Structure Analysis, 2nd ed.; Oxford University Press: New York, NY, USA, 2010; ISBN 9780195336047.

77. Castañar, L.; Sistaré, E.; Virgili, A.; Williamson, R.T.; Parella, T. Suppression of phase and amplitude J(HH) modulations in HSQC experiments. Magn. Reson. Chem. 2015, 53, 115-119. [CrossRef]

78. Koźmiński, W.; Nanz, D. Sensitivity Improvement and New Acquisition Scheme of Heteronuclear Active-Coupling-Pattern-Tilting Spectroscopy. J. Magn. Reson. 2000, 142, 294-299. [CrossRef]

79. Alabugin, I.V. Probing Stereoelectronic Effects with Spectroscopic Methods. In Stereoelectronic Effects: A Bridge Between Structure and Reactivity; Hoboken, N.J., Ed.; John Wiley \& Sons, Ltd.: Chichester, UK, 2016; pp. 1-7, ISBN 9781118906378.

80. Hameed, R.; van Mourik, T.; Khan, A. ${ }^{13} \mathrm{C}-{ }^{1} \mathrm{H}$ coupling constants as a conformational tool for structural assignment of quinic and octulosonic acid. J. Mol. Model. 2018, 24, 324. [CrossRef]

81. Ellman, G.L.; Courtney, K.D.; Andres, V.; Featherstone, R.M. A new and rapid colorimetric determination of acetylcholinesterase activity. Biochem. Pharmacol. 1961, 7, 88-95. [CrossRef]

82. Orhan, I.E.; Jedrejek, D.; Senol, F.S.; Salmas, R.E.; Durdagi, S.; Kowalska, I.; Pecio, L.; Oleszek, W. Molecular modeling and in vitro approaches towards cholinesterase inhibitory effect of some natural xanthohumol, naringenin, and acyl phloroglucinol derivatives. Phytomedicine 2018, 42, 25-33. [CrossRef] [PubMed]

83. Ślusarczyk, S.; Senol Deniz, F.S.; Woźniak, D.; Pecio, Ł.; Pérez-Sánchez, H.; Cerón-Carrasco, J.P.; Stochmal, A.; den-Haan Alonso, H.; Matkowski, A.; Orhan, I.E. Selective in vitro and in silico cholinesterase inhibitory activity of isoflavones and stilbenes from Belamcandae chinensis rhizoma. Phytochem. Lett. 2019, 30, 261-272. [CrossRef]

84. Rosenberry, T.; Brazzolotto, X.; Macdonald, I.; Wandhammer, M.; Trovaslet-Leroy, M.; Darvesh, S.; Nachon, F. Comparison of the Binding of Reversible Inhibitors to Human Butyrylcholinesterase and Acetylcholinesterase: A Crystallographic, Kinetic and Calorimetric Study. Molecules 2017, 22, 2098. [CrossRef] [PubMed]

85. Wagner, H.; Bladt, S. Plant Drug Analysis: A Thin Layer Chromatography Atlas, 2nd ed.; Elgazzar, A.H., Ed.; Springer: Berlin/Heidelberg, Gemany; New York, NY, USA, 2001; ISBN 3-540-58676-8.

86. Tyburn, J.-M.; Coutant, J. TopSpin ERETIC 2; Bruker Corporation: Billerica, MA, USA, 2015.

87. Burlec, A.F.; Pecio, Ł.; Mircea, C.; Cioancă, O.; Corciovă, A.; Nicolescu, A.; Oleszek, W.; Hăncianu, M. Chemical Profile and Antioxidant Activity of Zinnia elegans Jacq. Fractions. Molecules 2019, $24,2934$. [CrossRef]

88. Kozachok, S.; Pecio, Ł.; Orhan, I.E.; Deniz, F.S.S.; Marchyshyn, S.; Oleszek, W. Reinvestigation of Herniaria glabra L. saponins and their biological activity. Phytochemistry 2020, 169, 112162. [CrossRef]

89. Frisch, M.J.; Trucks, G.W.; Schlegel, H.B.; Scuseria, G.E.; Robb, M.A.; Cheeseman, J.R.; Scalmani, G.; Barone, V.; Petersson, G.A.; Nakatsuji, H.; et al. Gaussian 16, Rev. A.03; Gaussian, Inc.: Wallingford, CT, USA, 2016.

90. Cheung, J.; Rudolph, M.J.; Burshteyn, F.; Cassidy, M.S.; Gary, E.N.; Love, J.; Franklin, M.C.; Height, J.J. Structures of Human Acetylcholinesterase in Complex with Pharmacologically Important Ligands. J. Med. Chem. 2012, 55, 10282-10286. [CrossRef]

91. Luo, H.-J.; Wang, J.-Z.; Huang, N.-Y.; Deng, W.-Q.; Zou, K. Induced-fit docking and virtual screening for 8-hydroxy-3-methoxy-5H-pyrido [2,1-c] pyrazin-5-one derivatives as inducible nitric oxide synthase inhibitors. J. Chem. Pharm. Res. 2014, 6, 1187-1194.

Sample Availability: Samples of the compounds are not available from the authors. 